\title{
Grounded Theory als Instrument der Weltpolitikforschung. Die Rekonstruktion außenpolitischer Kultur als Beispiel
}

\author{
Ulrich Roos ${ }^{1}$
}

\section{Einleitung}

In diesem Beitrag beschreibe ich, auf welche Art und Weise ich gegenwärtig sozialwissenschaftliche Forschung betreibe. Ich verfolge dabei das Ziel, Ihnen so klar und deutlich wie es mir möglich ist, zu erklären, wie ich meine Forschungspraxis gestalte. Sozialwissenschaftliche Forschung, so meine Überzeugung, ist kein Buch mit sieben Siegeln. Ganz im Gegenteil: Rekonstruktive Methoden basieren auf zwei grundlegenden Operationen des menschlichen Geistes: Denken und Ordnen. Wohl sämtliche wissenschaftlichen Methoden greifen auf diese beiden Fähigkeiten des Menschen zurück. Wer denken und ordnen kann, so glaube ich, kann wissenschaftlich arbeiten. Auf den folgenden Seiten werde ich darüber schreiben, wie ich mein Denken ordne und wie ich über das Geordnete denke.

Dass ich gleich im ersten Satz die Einschränkung formuliert habe, nur beschreiben zu wollen, wie ich, gegenwärtig ' forsche, ist keine Koketterie. Wie ich zukünftig über Forschung denken werde, weiß ich heute noch nicht und es würde mich verzweifeln lassen, versuchte ich genau nachzuzeichnen, wie sich im Laufe der Zeit mein Denken über Methode und Forschung verändert hat. Wer sich auf Forschung einlässt, verändert sein Denken - auch und gerade mit Blick auf Methoden - fortlaufend.

Aus diesem Grund wird der vorliegende Beitrag auch nicht erläutern, wie ich im Rahmen meines vor neun Jahren begonnenen und vor fünf Jahren abgeschlossenen Dissertationsvorhabens geforscht habe, sondern wie ich gegenwärtig, im Rahmen eines aktuellen Forschungsvorhabens forsche, da sich meine methodische Vorgehensweise in den vergangenen Jahren sukzessive verändert hat. Die Dissertation eröffnete mir dabei zunächst die Möglichkeit, über einen Zeitraum von vier Jahren an

1 Ich bedanke mich bei den anonymen GutachterInnen, den TeilnehmerInnen des Forschungskolloquiums des Lehrstuhls für Friedens- und Konfliktforschung der Universität Augsburg, den TeilnehmerInnen der Grounded Theory-Seminare des MA-Sozialwissenschaftliche Konfliktforschung sowie der Angewandte Methoden-Seminare des BA-Sozialwissenschaften der Universität Augsburg und natürlich meinem Freund und Herausgeberkollegen Ulrich Franke für die an den vorgängigen Versionen des Manuskripts geäußerte Kritik. 
nichts anderem zu arbeiten als an einer einzigen Fragestellung: Welche grundlegenden Handlungsregeln leiten deutsche Außenpolitik an und wie haben sich diese Handlungsregeln zwischen 1990 und 2007 entwickelt? In dieser Zeit erfuhr ich, vor welch`vielschichtigen Schwierigkeiten sozialwissenschaftliche Forschung steht und musste die immer wieder sich einstellenden Zweifel an der eigenen Vorgehensweise bearbeiten, um das Forschungsvorhaben beginnen, vorantreiben und abschließen zu können. Genauso wertvoll sind jedoch jene Erfahrungen, die ich im Rahmen von Lehrforschungsprojekten, Forschungs- und Methodenseminaren als Dozent sammeln darf. Es ist das eine, für die eigene methodische Vorgehensweise verantwortlich zu sein, etwas ganz anderes, als Ratgeber und in gewisser Weise auch Mitverantwortlicher die Forschungsvorhaben Dritter zu begleiten.

Um Ihnen meine methodische Vorgehensweise möglichst konkret erläutern $\mathrm{zu}$ können, lade ich Sie auf den folgenden Seiten in mein ,Labor' ein und stelle so oft wie möglich Bezüge zu einem meiner laufenden Forschungsvorhaben her, so dass wir gemeinsam keine, Trocken-Schwimmübungen' betreiben müssen, sondern miteinander ins Wasser gehen können, um die Schwimmtechniken nicht bloß zu simulieren, sondern unmittelbar beobachten zu können. Wir lernen oftmals zunächst auf Basis von Imitation und Sie sollten sich vollkommen frei fühlen, alles, was Ihnen sinnvoll erscheint, zu imitieren und in der Folge experimentell Ihren ganz eigenen Stil zu entdecken. Letztlich wird wohl jede Forscherin ihren eigenen Forschungsstil entwickeln - mehr als eine Anregung kann der hier präsentierte Ansatz daher nicht bedeuten.

Im nachfolgenden, zweiten Abschnitt stelle ich zunächst eine Forschungsfrage vor, an der ich momentan arbeite und erläutere die epistemologischen und ontologischen Prämissen, auf der diese fußt. Sie erfahren, was mein Untersuchungsgegenstand ist, wie ich über dessen Beschaffenheit denke und aus welcher theoretischen Perspektive ich diesen betrachte. Ich erläutere kurz die Forschungsfrage, so dass Sie verstehen, worin genau mein Forschungsinteresse gründet und was mich umtreibt. Insofern meine Art und Weise, über Methodologie und Methode zu denken, stark von den Vorarbeiten Dritter beeinflusst wurde, werde ich in der gebotenen Kürze eine Selbstverortung in einer breiteren ,Forschungstradition“ vornehmen, so dass Sie eventuell weitere Anregungen für Ihre zukünftige Lektüre zum Thema ,Methode“ erhalten. Der erste Abschnitt schließt mit einer kurzen Reflexion zum Thema ,Gütekriterien' rekonstruktiver Weltpolitikforschung. Dort werde ich meine Position zur Frage nachzeichnen, was Sozialwissenschaft eigentlich zu leisten vermag und wie ich es ,mit der Wahrheit halte'.

Im dritten Abschnitt erkläre ich, wie ich zurzeit forsche. Dabei orientiere ich mich an dreizehn Fragen, von denen ich glaube, dass jede Forscherin hierauf Antworten formulieren sollte. 


\section{Von Erkenntnis, Sein und Wissenschaft. Oder: Epistemologische, ontologische und methodologische Überzeugungen}

Sämtliche in diesem Beitrag dargelegten theoretischen, methodologischen und methodischen Überzeugungen verdanke ich den Vorarbeiten Dritter. Dieser Gedanke mutet trivial an, ist mir aber wichtig: Ohne Dritte und deren Vorarbeit gäbe es gar keine Sprache, in der ich mich auszudrücken vermag, gäbe es keine Bücher, keine Theorien, die ich lesen und auf die ich mich beziehen könnte. Mein Denken und alle Überzeugungen sind Resultat der Auseinandersetzung mit den Ideen meiner Vor- und Mitwelt. Soweit mir eine solche Selbstreflexion möglich ist, möchte ich jedoch den Einfluss einiger Arbeiten auf die hier von mir präsentierte Art und Weise, über das Soziale und die Methoden seiner Erforschung zu denken, besonders hervorheben, um jenen Leserinnen, die ihre Lektüre zum Thema rekonstruktive Forschung fortsetzen möchten, einige Anregungen zu liefern. Die meisten der hier in einem ,raschen Galopp` nur kurz erwähnten Konzepte und Ideen werden in später folgenden Abschnitten erneut aufgegriffen und erörtert. Wer sich also mit Blick auf weitere Lektüre nicht ,anregen' lassen möchte, kann die folgende Seite auch überspringen.

Stark beeinflusst hat mich die Auseinandersetzung mit dem Grounded TheoryVerfahren, wie es von Anselm Strauss nach seiner ,Trennung ' von Barney Glaser mit dem er dieses Verfahren zunächst gemeinsam erarbeitet hatte (Glaser/Strauss 1967) - weiterentwickelt wurde (Strauss 1987; Strauss/Corbin 1998). Dabei wurde Strauss (1916-1996) besonders vom Symbolischen Interaktionismus Herbert Blumers (1900-1987) und den Ideen der klassischen Pragmatisten, allen voran von George Herbert Mead (1863-1931), aber auch von John Dewey (1859-1952) geprägt (Strauss 1993: 4-11). Sollte der Leser sich weiterhin mit Grounded Theory in der Lesart von Anselm Strauss beschäftigen wollen, seien Jörg Strübing (2004) und die Beiträge im Sage Handbook (Bryant/Charmaz 2010) als Einstiegslektüre empfohlen. Die zentralen Gedankengänge von Strauss finden sich in Strauss (1994 und 2004 a) sowie in Strauss/Corbin (1998). Sein grundlegendes handlungstheoretisches Werk legte er mit „Continual Permutations of Action“ erst 1993 vor. Der Lektüre seiner Texte verdanke ich neben all den methodologischen und methodischen Anregungen auch den bedeutenden Ratschlag, Forschung zwar stets als Arbeit zu verstehen, doch dabei eine Haltung einzunehmen, die es ermöglicht, auch Freude an dieser Tätigkeit zu haben.

John Deweys Arbeiten zur Öffentlichkeit und ihren Problemen (Dewey [1927] 1991) und seine Ausführungen darüber, ,wie wir denken“ (Dewey [1910] 1997) haben meine Art und Weise, Gesellschaft zu begreifen und mein Forschungsverständnis nachhaltig geprägt. Ich verdanke ihm die theoretische Figur der ,Struktur kollektiven Handelns' und sympathisiere mit seiner starken Überzeugung, dass alltägliches Pro- 
blemlösen und wissenschaftliche Forschung keine kategorial verschiedenen Tätigkeiten darstellen.

Die Interpretation der Vorarbeiten von Charles Sanders Peirce durch Ulrich Oevermann $(1991,1996)$ haben mich für die Bedeutung der Sequenzialität menschlicher Interaktion sensibilisiert, was gemeinsam mit Meads Modell vom „I“ und „me“ (Mead [posthum 1934] 1974) mein Verständnis der sozialen Identität des und der Menschen beeinflusst hat. Schließlich hat Charles Sanders Peirce mit dem Begriff der Handlungsregel (Peirce [1878] 1992: 129) und dem Konzept der finalen Gründe (Peirce [1902] 1998: 120), mein Modell des Sozialen maßgeblich orientiert, genauso wie seine Interpretation des aristotelischen Kausalitätsverständnisses und die sogenannte pragmatistische Maxime (Peirce [1905] 1998: 338). Schließlich verdankt ihm die moderne Wissenschaft die Neu-Entdeckung des abduktiven Schließens, das er ebenfalls unter Rückgriff auf Aristoteles in die Wissenschaftstheorie eingeführt hat (Peirce [1903] 1965: 106 (5.171)).

Wenn ich also nachfolgend einige Gedanken bezüglich der Möglichkeiten der Erforschung von Weltpolitik formuliere, dann wäre dies ohne die Vorarbeiten dieser und zahlreicher hier zu Unrecht nicht erwähnter Dritter gar nicht möglich gewesen. Ich selbst verstehe meine Art und Weise zu forschen als eine Modifikation des Grounded Theory-Verfahrens in Anlehnung an Anselm Strauss (vgl. hierzu auch den Beitrag von Ursula Jasper in diesem Band). Obwohl ich nicht mehr länger - wie noch in meinem Dissertationsprojekt - mit den Begriffen des offenen, axialen und selektiven Kodierens arbeite, nicht mehr in substantielles und theoretisches Kodieren unterscheide, weil für mich alles Kodieren immer theoretisch ist und ich diese Trennung daher als unglücklich empfinde und ich noch nie nach nur einer Schlüsselkategorie geforscht habe, verstehe ich meine Vorgehensweise noch immer als eine Variante der Grounded Theory (Roos 2012). Und dies weniger, um mit dem ,GT'-Label Werbung für den eigenen Ansatz zu machen, sondern weil ich glaube, dass mich die Methodologie von Strauss so sehr beeinflusst hat, dass es ganz im Gegenteil unredlich wäre, diese Tatsache nicht klar zum Ausdruck zu bringen. Und bei allen Modifikationen halte ich an den drei von Strauss als Essentials seiner Arbeitsweise hervorgehobenen Konzepten i) permanenter Vergleich, ii) theoretical sampling und iii) theoretisches Kodieren fest (Strauss 2004 b).

Die bis hierher erfolgte, kurze ,Würdigung ' der Gedanken Dritter erscheint mir vor allen Dingen auch deswegen notwendig, da ich in den anschließenden Abschnitten des Textes ,ganz bei mir bleiben und Verweise auf andere, ähnliche Arbeiten und Ansätze nur sporadisch einflechten werde. Ohne diese wären meine ,eigenen“ Überzeugungen, Techniken und Praktiken in dieser Form möglicherweise jedoch nie entstanden.

Wer, Weltpolitik' erforschen möchte, wird dies auf Grundlage spezifischer theoretischer Vorannahmen mit Blick auf die Beschaffenheit des jeweils spezifischen 
Untersuchungsgegenstands tun. Jeder Mensch erarbeitet, so behaupte ich, im Laufe seines Lebens eine ganz eigene Theorie des Sozialen, die sein eigenes Handeln orientiert, indem ihm durch diese Theorie soziale Interaktion verständlich gemacht wird. Ich bin davon überzeugt, dass am Anfang jeder sozialwissenschaftlichen Arbeit die Explikation dieser oftmals in der Latenz verbleibenden sozial- und handlungstheoretischen Prämissen des forschenden Subjekts stehen sollte. Erst auf Basis einer solchen Reflexion der theoretischen Überzeugungen des Forschers werden die Zielsetzung der Forschungsfrage und der Sinn der darin verwendeten Begrifflichkeiten, sowohl für den Forscher selbst als auch für Dritte, verständlich.

Zur Zeit beschäftige ich mich gemeinsam mit meinem Kollegen Timo Seidl mit der Frage, welche grundlegenden Handlungsregeln und finalen Gründe die NamibiaPolitik der Fraktionen des Deutschen Bundestags und der Bundesregierung seit 1989/90 bestimmen und welche Elemente von Kontinuität und Wandel sich bezüglich der darin repräsentierten außenpolitischen Kultur Deutschlands identifizieren lassen.

Um die Anlage und Stoßrichtung dieser Forschungsfrage verstehen zu können, bedarf es offensichtlich i) einer Explikation der Konzepte ,Handlungsregel' und ,finale Gründe', ii) eines Außenpolitikmodells und iii) einer theoretischen Figur, die ,Entwicklung' definiert. Anders gesprochen: Die oben stehende Forschungsfrage wird erst vor dem Hintergrund des von mir präferierten pragmatistischen Analyserahmens besser verständlich, der nachfolgend in der gebotenen Kürze dargestellt werden soll. ${ }^{2}$ Diese Vorarbeit, die Explikation der theoretischen Modelle des Forschers, steht für mich am Anfang jeder sozialwissenschaftlichen Forschung.

\subsection{Formaltheoretische Vorannahmen}

Der erklärungsbedürftige Begriff der Handlungsregel stellt in der hier behandelten Forschungsfrage die zentrale formaltheoretische Figur des entworfenen Analyserahmens dar. Diese Idee geht auf die Vorarbeiten des klassischen Pragmatisten Charles Sanders Peirce zurück, der Überzeugungen als Handlungsregeln (»belief is a rule for action«) begriffen hat (Peirce [1878] 1992: 129).

2 Pragmatistisch inspirierte Beiträge nehmen in den Internationalen Beziehungen inzwischen an Zahl und Popularität zu (Wagner et al. 2006; Bauer/Brighi 2009; Hellmann 2009, 2010; Friedrichs/Kratochwil 2009; Franke/Roos 2010; Franke/Weber 2012), gleichwohl sind davon angeleitete Forschungsarbeiten - ganz zu schweigen von Präsentationen hierdurch erarbeiteter Befunde - nach wie vor eher selten aufzufinden (vgl. jedoch Baumann 2006; Hellmann 2006; Hellmann et al. 2008, Hellmann/Roos 2007; Franke 2010; Roos 2010, 2012; Hofferberth 2012; Jasper 2014). 
Jedes Handeln wird, so die Annahme, von erfahrungsgesättigten Handlungsregeln angeleitet. Dabei können Akteure aufgrund ihrer je spezifischen Erfahrungen unterschiedliche Handlungsregeln befolgen, doch diese nicht voluntaristisch, ex nihilo, aus sich selbst heraus schöpfen. Alle Handlungsregeln, so unterschiedlich sie sein mögen, sind Ergebnis sozial vermittelter Lernprozesse, die durch den Gebrauch gemeinsam geteilter Sprachzeichen kommuniziert und so intersubjektiv nachvollzogen werden können. Insofern die soziale Interaktion aller Akteure fortlaufend intendierte und nicht-intendierte Auswirkungen zeitigen, das Soziale daher genuin prozesshaft verläuft und für fortlaufende Veränderungen des größeren Bedeutungsuniversums und der gemeinsam geteilten Symbolwelt sorgt, geraten Überzeugungen immer wieder unter Bewährungsdruck. Mit mannigfaltigen Reaktionen ihrer Umwelt konfrontiert, hinterfragen bzw. reflektieren die Akteure, egal ob bewusst oder unbewusst, ihre jeweiligen Handlungsregeln fortlaufend - und zwar umso stärker und schneller, je krisenhafter die Reaktion der Umwelt auf das eigene Handeln empfunden wird. Dementsprechend modifizieren sie ihre Handlungsregeln. Die seitens einer Struktur kollektiven Handelns (zum Begriff der Struktur kollektiven Handelns, siehe Dewey [1927] 1991: 190; Franke/Roos 2010; Roos 2010), wie z.B. der Regierung oder des Parlaments der Bundesrepublik Deutschland, verfolgten (außenpolitischen) Handlungsregeln weisen aus diesem Grund zu jedem Zeitpunkt sowohl Elemente von Kontinuität als auch Wandel auf, da sich zu jedem gegebenen Zeitpunkt einige Handlungsregeln in einer Bewährungskrise befinden und umstritten sind, während andere Handlungsregeln als bewährt gelten und als Routine ablaufen. Die Handlungsregeln der Akteure unterliegen also einer fortlaufenden Entwicklung, wobei Wandel und Kontinuität miteinander Hand in Hand gehen. Das Reservoir der handlungsleitenden Regeln einer Struktur kollektiven Handelns verstehe ich als dessen (politische) Kultur.

Als Struktur kollektiven Handelns bezeichne ich solche sozialen Institutionen, die das kollektive Handeln mehrerer Personen mit dem Ziel der Regulierung der Folgen der Auswirkungen des kollektiven Handelns ermöglichen sollen. Strukturen kollektiven Handelns (SKH), wie zum Beispiel ein Staat, eine internationale Organisation, eine Familie oder ein Verein werden als in Prozesse eingebettet verstanden, deren Gehalt aus verschiedenen Handlungsregeln und finalen Gründen besteht, die über Identität und Ziele der SKH bestimmen.

Die in der oben stehenden Fragestellung erwähnten, finalen Gründe' stellen nun jene Handlungsregeln dar, welche die Ziele der Handelnden bestimmen und für die wir uns besonders interessieren sollten, wenn wir versuchen, soziale Interaktion zu erklären. Peirce definiert einen finalen Grund als die idealtypischen Vorstellungen der Handelnden mit Blick auf ein mögliches Endstadium eines Prozesses (auf die dieser hin tendiert), indem das Handeln der Akteure dieses Endstadium zu erreichen versucht. Für Peirce lassen sich soziale Prozesse daher ohne Berücksichtigung finaler 
Gründe nicht erklären, beziehungsweise sie würden jeden Sinn entbehren und könnten dann nicht einmal mehr als Chaos bezeichnet werden (Peirce [1903] 1965: 97 (1.220)). ${ }^{3}$ Für ihn besteht zwischen der Wirkursache (,efficient cause“), dem Effekt dieser Wirkursache, dem finalen Grund und dem realisierten Ende des Prozesses folgende, auf den zweiten Blick formaltheoretisch banale Verbindung:

Die Wirkursache als Handlung (A) wird durch den gewünschten möglichen Ausgang des Handlungsprozesses $\left(\mathrm{C}^{\prime}\right)$ bestimmt. Von der Handlung (A) geht eine Wirkung (B) aus, die ein Ergebnis (C) zeitigt, das tendenziell dem gewünschten möglichen Ausgang $\left(\mathrm{C}^{\prime}\right)$ entspricht. Der finale Grund $\left(\mathrm{C}^{\prime}\right)$ ist insofern bloß eine Idee des möglichen Ausgangs eines Handlungsprozesses; sie ist eine „Möglichkeit“ (Hulswit 2002: 81). Aus der Analyse finaler Gründe geht hervor, welche generellen Prinzipien (als Handlungsregeln) die Tendenzen und Ziele eines sozialen Prozesses bestimmen.

Die propositionalen Gehalte einer Überzeugung als Handlungsregel lassen sich, so lautet eine weitere Annahme, unter Rückgriff auf interpretativ verfahrende Methoden, aus den protokollierten Spuren von Interaktion rekonstruieren (Oevermann 1991: 295). Wer nun die Entwicklung - verstanden als Gleichzeitigkeit von Kontinuität und Wandel - des (außenpolitischen) Handelns Deutschlands (oder eines anderen Staates oder einer anderen Struktur kollektiven Handelns wie z.B. einer Familie, einem Verein oder einem Freundeskreis) z.B. bezogen auf die deutsche Namibiapolitik erklären möchte, der muss, falls ihm diese theoretischen Annahmen plausibel erscheinen, die dem Handeln jeweils zugrundeliegenden Handlungsregeln im Zeitverlauf dechiffrieren. Aus dieser theoretischen Perspektive betrachtet, gilt jedes Sprechen immer bereits als Handeln, da es immer schon Reaktion auf Interaktion darstellt und auch selbst Reaktionen auslöst. ${ }^{4}$

In diesem Zusammenhang muss ich Sie - um meine methodische Vorgehensweise besser verständlich zu machen - mit einer weiteren theoretischen Figur vertraut machen, die ebenfalls von Peirce formuliert und als sogenannte „Pragmatistische Maxime" bekannt wurde:

„Consider what effects that might conceivably have practical bearing you conceive the object of your conception to have. Then your conception of those effects is the WHOLE of your conception of the object" (Peirce [1905] 1998: 338; Hervorhebung im Original).

3 ,Efficient causation is that kind of causation whereby the parts compose the whole; final causation is that kind of causation whereby the whole calls out its parts. Final causation without efficient causation is helpless...efficient causation without final causation, however is worse than helpless, by far; it is mere chaos; and chaos is not even so much chaos, without final causation; it is blank nothing" (Peirce [1903] 1965: 97 (1.220)).

4 Zum Verhältnis von Akteur, Struktur und Prozess aus pragmatistischer Perspektive vgl. Franke/Roos (2010) und Roos (2010: 48-77). 
Aus diesem einfachen Satz resultieren ganz erhebliche methodologische Konsequenzen. Ich verstehe Peirce so, dass wir die Identität eines (sozialen) Gegenstands, gleich ob es sich dabei um eine Person, einen Staat, eine Partei, einen Tisch oder die Vereinten Nationen handelt, über die ihm zugeschriebenen Wirkungen begreifen können. Was deutsche Außenpolitik ist, was die Namibiapolitik der deutschen Regierung ist, lässt sich anhand der ihr zugeschriebenen Bedeutungen rekonstruieren. Dabei stehen Selbstbeschreibungen und Fremdbeschreibungen gleichberechtigt nebeneinander (obgleich es wahrscheinlich allzu philosophisch sein dürfte, über die Selbstbeschreibungen eines Tisches nachzudenken). Beide Beschreibungstypen stellen Reaktionen, Wirkungen, Interpretationen auf bisherige Erfahrungen mit dem jeweiligen Gegenstand dar.

Über ihre, eigene“ Identität und Bedeutung entscheiden Menschen und Kollektive nicht allein, sondern unsere Identität und Bedeutung ist zumindest genauso sehr von den Beschreibungen, Interpretationen und Reaktionen unserer Umwelt bestimmt. Das bedeutet, dass ich mich zur Beantwortung der oben aufgeworfenen Forschungsfrage auf die Suche nach den Wirkungen deutscher Namibiapolitik machen muss. Dabei stellen auch sämtliche jemals aufgestellten wissenschaftlichen Theorien bezüglich der Identität der außenpolitischen Kultur Deutschlands Effekte deutscher Außenpolitik dar, genauso wie alle anderen darauf reagierenden Äußerungen und Handlungen Dritter, der internationalen Umwelt etwa. Wer eine Theorie der Identität der außenpolitischen Kultur Deutschlands formulieren möchte, darf nicht alleine die (Sprech-)Handlungen deutscher Außenpolitiker analysieren, sondern sollte - und hierin liegt der eigentliche Clou der pragmatistischen Maxime - unbedingt die Reaktionen Dritter auf diese Außenpolitik als Datenmaterial mitberücksichtigen.

Die allem sozialen Handeln zugrundeliegenden Überzeugungen als Handlungsregeln der Akteure lassen sich durch die Interpretation von textförmig protokollierten Spuren sozialer Interaktion rekonstruieren. Doch was genau sind ,textförmig protokollierte Spuren sozialer Interaktion"? Die Rekonstruktion stützt sich nicht allein auf Sprechakte, sondern auf sämtliche Typen von Spuren, die der Untersuchungsgegenstand hinterlassen hat. Die zu untersuchende Lebenspraxis hinterlässt möglicherweise Text-, Video- oder Audioprotokolle, provoziert entsprechend dokumentierte Reaktionen seitens Dritter oder hinterlässt andere Spuren ihres Handelns. So lässt sich die Identität der außenpolitischen Kultur Deutschlands nicht allein über die Analyse regierungsamtlicher Texte und Reden rekonstruieren, sondern ganz im Sinne der oben erörterten pragmatistischen Maxime auch bzw. ganz besonders aufgrund der Reaktionen Dritter auf deutsche Außenpolitik bzw. aufgrund der seitens Dritter deutscher Außenpolitik zugesprochenen Eigenschaften. Doch auch die Analyse von Handlungsformen jenseits offizieller Sprechakte dient unseren Analysen als Material.

Wer z.B. etwas über die Beschaffenheit des Ost-West-Konfliktes in Erfahrung bringen möchte und diesbezüglich an einer Theorie arbeitet, wird die Stationierung 
von Mittelstreckenraketen seitens der UdSSR auf Kuba ebenfalls als Datenmaterial, das es zu interpretieren gilt, in die Rekonstruktion integrieren, auch wenn diese Form des Handelns gerade nicht in Form eines regierungsoffiziellen Sprechaktes erfolgte. Im Rahmen unserer Forschungsarbeit zu den deutsch-namibischen Beziehungen interessieren Timo Seidl und ich uns nicht ausschließlich für Festtagsreden, sondern auch dafür, welche bilateralen Verträge geschlossen, welche Kooperationsprogramme initiiert wurden, mit welchem Budget diese versehen worden sind, welche Repräsentanten der Bundesregierung wie oft und wie lange zu Staatsbesuchen nach Namibia reisen, welche Visa-Absprachen getroffen, welche bedeutenden ökonomischen Vorhaben gemeinsam realisiert wurden usw.

Meiner Meinung nach erfolgt die Auswahl des Datenmaterials in der Weltpolitikforschung aufgrund zweier Vorurteile oftmals suboptimal: Eine von mir hier nur idealtypisch skizzierte Gruppe von Forschern hält generell Sprechakte für ungeeignetes Material, da ,bloßes Reden“ kein Handeln sei und interessiert sich aus diesem Grund oftmals nur für vermeintlich ,harte Fakten' wie Außenhandelsbilanzen, Volumina von Wirtschaftskooperationen, Militärbudgets, Dauer und Frequenz militärischer Auseinandersetzung usw. Eine zweite Gruppe von Forschern berücksichtigt bei ihren Arbeiten hingegen ausschließlich Sprechakte unter Verweis auf die sozialkonstruktivistische Idee, der Sinn von Realität würde durch Sprechen konstruiert. Beide Vorgehensweisen verschließen sich meines Erachtens ohne Not jeweils für bestimmte Typen protokollierter Spuren sozialen Handelns. Selbstverständlich besteht zwischen einem Raketenangriff und einer vor den UN gehaltenen Rede eine Differenz mit Blick auf das Handlungsmedium, weswegen es sich um unterschiedliche Handlungsformen handelt. Sollten wir uns für die Identität eines bestimmten Phänomens, sei es eine Struktur kollektiven Handelns oder eines Handlungsprozesses interessieren, besteht jedoch kein Grund, prinzipiell auf bestimmte Typen von Handlungsspuren zu verzichten. Alle Typen von Spuren sozialen Handelns verbindet zudem, dass sie sich uns ,textförmig' erschließen. Das heißt, wir schreiben den beobachteten Spuren sozialer Praxis Bedeutung und Sinn zu, wir verbinden in unserer Wahrnehmung und Interpretation mit Bildern, Tönen oder einem Raketenabschuss Begriffe und lesen diese als Text.

Auf Grundlage dieser Explikation meiner theoretischen Prämissen sollte die mich beschäftigende Forschungsfrage nach den grundlegenden Handlungsregeln und finalen Gründen der Namibiapolitik der Fraktionen des Deutschen Bundestags und der Bundesregierung und deren Entwicklung seit 1989/90 besser verständlich sein als zuvor. 


\subsection{Epistemologische Reflexion}

Neben die Explikation der ontologischen Prämissen sollte meines Erachten eine epistemologische Reflexion treten, die Antworten auf die Frage formuliert, welche Qualität die erarbeiteten Befunde der Forschung aufweisen sollen und wie der Forscher es mit der Wahrheit hält. Mit Blick auf Letztere vertrete ich derzeit eine Position, die diese Idee nicht absolut setzt, sondern als Resultat gesellschaftlicher Deliberation versteht. Wahr ist, was als wahr gilt. Die Reichweite der Geltungsansprüche wissenschaftlicher Befunde erscheint in dieser Perspektive als abhängig vom Grad der Zustimmung der scientific community. Ich verstehe Forschung nicht als das Schürfen in den Bergwerkstollen der Wahrheit, mit dem Ziel, Stück für Stück dieses kostbaren Gutes für alle Zeit zutage zu fördern. Wenn etwas ,wahr' sein soll, dann kann es sich unmöglich zu einem späteren Zeitpunkt als unwahr entpuppen. Insofern ich jedoch angesichts der endlichen Vernunft des Menschen nicht an die Möglichkeit glaube, dass uns die Einsicht in solch ewigen Wahrheiten möglich ist, halte ich absolute Wahrheit für eine Illusion. Alle wissenschaftlichen Wahrheiten - selbst die von Menschen formulierten Behauptungen mit Blick auf die sogenannten Naturgesetze - müssen in dieser Perspektive als grundsätzlich fallibel gedacht werden. Wahrheiten sind Hypothesen, die in der scientific community für einen unbestimmten Zeitraum als plausibel gelten. Es handelt sich um Theorien, Vermutungen, Behauptungen, Hypothesen, die als besonders plausibel (,plausibilis' bzw. ,plaudere' stehen für ,beifallswürdig' bzw. ,Beifall klatschen') wahrgenommen werden, große Suggestionskraft entwickeln, sich besonders passförmig in andere Theoreme einfügen und die es ermöglichen, die Phänomene der wahrgenommenen Realität(en) zu erklären.

Wenn generell das soziale Leben als aufgrund konkurrierender Überzeugungen, Werte, Ideen und Normen ausgetragener Kampf um Deutungshoheit und Entscheidungsmacht zu verstehen ist, dann gilt dies erst recht für die Sozialwissenschaften, die in diesem Verständnis einen eminent politischen Beruf darstellen. Denn aus meiner Sicht besteht die Funktion rekonstruktiver Sozialforschung ja eben gerade darin, die bezogen auf eine bestimmte gesellschaftliche Herausforderung miteinander um Geltung ringenden politischen Überzeugungen je für sich zu rekonstruieren und z.B. im Sinne einer kritischen Diskursforschung so klar zu beschreiben, dass die zwischen den umkämpften Alternativen bestehenden Unterschiede von der größeren Gesellschaft nachvollzogen werden können. Durch die Rekonstruktion der verschiedenen Problembeschreibungen und Lösungsvorschläge wird ein Denken in Alternativen begünstigt, das wohl den Kern öffentlicher, demokratischer Deliberation darstellt. Insofern eine solche Forschung sich nicht wertfrei vollziehen kann, sondern aufgrund der politischen Präferenzen des forschenden Subjekts zumindest implizit Wertungen vornimmt, muss diese Tatsache seitens der Leserin der wissenschaftlichen Befunde immer schon mitgedacht werden. 
Verbinden wir nun diesen Gedanken mit der ablehnenden Haltung bezüglich ,der einen Wahrheit‘, ergibt sich daraus ein Wissenschaftsverständnis, welches von der Idee geprägt ist, möglichst viele verschiedene Forscherinnen mögen sich mit einem relevanten Thema je aus ihrer Perspektive auseinandersetzen und eine Rekonstruktion - in Form einer Theorie, der Erklärung eines Sachverhalts also - vorlegen. Die solcherart miteinander konkurrierenden oder harmonierenden Rekonstruktionen können dann verglichen werden, so dass durch das Identifizieren von Gemeinsamkeiten und Unterschieden zwischen den vorliegenden Theorien ein produktiver, akademischer Streit im besten Sinne entstehen wird, der auch der gesellschaftlichen Willensbildung als Material dienen kann.

Ist Sozialforschung, ist rekonstruktive Weltpolitikforschung dann also letztlich der methodischen Beliebigkeit, der Relativität und Subjektivität ausgesetzt? Ja und nein. Einerseits ist es unmöglich, ewig gültige Gütekriterien zu identifizieren, anhand derer wir über die Qualität wissenschaftlicher Befunde urteilen können, denn auch diese Kriterien stellen ja bloß soziale Konventionen dar, die ohne Letztbegründung auskommen müssen. Reliabilität, interne und externe Validität, Objektivität, Repräsentativität usw. sind menschengemachte Beurteilungskriterien, die immer angreifbar bleiben werden. Aus Platzgründen kann ich meinen Standpunkt zum Gütekriteriendiskurs hier nicht wiederholen. Interessierte finden ihn im vierten Kapitel meines Buches zur deutschen Außenpolitik (Roos 2010: 90-99). Stattdessen möchte ich kurz jene Kriterien in der nachfolgenden Tabelle zusammenfassen, anhand deren ich inzwischen selbst die Qualität rekonstruktiv verfahrender Forschung bewerte. Es handelt sich dabei um:

\section{Qualitätskriterien rekonstruktiv verfahrender Forschung}

- Kohärenz und Konsistenz der generierten Theorie

- Intensität der Reflexion problematischer Auswahlentscheidungen

- Intersubjektive Nachvollziehbarkeit des Forschungsprozesses

- Sinnvolle Systematisierung des Daten- und Interpretationskorpus

- Umfang der Rekonstruktionsarbeit (Materialumfang)

- Intensität der Rekonstruktionsarbeit (Präzision und Dichte des theoretischen Kodierens)

- Transparenz mit Blick auf das Vorwissen und die Vorurteile des Forschers

Während die meisten dieser Kriterien entweder selbsterklärend sind bzw. auf den folgenden Seiten noch näher erörtert werden, gilt dies für den letztgenannten Punkt nicht. Ich versuche in meinen eigenen Forschungsprojekten die ,Transparenz mit Blick auf das Vorwissen bzw. die Vorurteile des Forschers` zu erhöhen, indem ich 
zu Beginn des Forschungsprozesses ein ausführliches Memo anfertige, ${ }^{5}$ in welchem ich darüber reflektiere, warum ich mich für die formulierte Forschungsfrage interessiere und welche Antworten auf diese Frage ich gegenwärtig für besonders plausibel erachte. Auf diese Weise schaffe ich einen systematischen Ort zur Selbstbeobachtung bezüglich der eigenen Vorurteilsstruktur. Dies unterstützt mich bei der Kodierarbeit, da ich beim Interpretieren besser begreife, wann ich meine subjektive Vorurteilsstruktur bediene oder Thesen formuliere, die mich selbst überraschen, da sie von diesen Vorannahmen abweichen. Möglicherweise unterstützt der solcherart gewährte, selbstverständlich ebenfalls subjektiv verzerrte Einblick (einige Überzeugungen werde ich wohl auch vor mir selbst, noch mehr vor der Öffentlichkeit verbergen) in meine Überzeugungen und den gegenwärtigen Hypothesenbestand auch die Leser der von mir später veröffentlichten Abschlussberichte bei ihrer Interpretation der vorgelegten Theorie.

Wie aber gestaltet sich die konkrete methodische Vorgehensweise einer sinnrekonstruierenden Weltpolitikforschung? Um dies zu zeigen, schließe ich diesen Abschnitt meines Beitrags mit einem Katalog von Leitfragen, die zu berücksichtigen den Forschungsprozess meines Erachtens unterstützt. Im dritten Abschnitt dieses Beitrags werde ich die von mir im Rahmen des Namibia-Projekts formulierten Antworten auf diese Fragen präsentieren

\section{Fragen rekonstruktiver Sozialforschung}

1. Wie lautet die Forschungsfrage? Nach welcher Art von Antwort verlangt diese Frage? Was bedeutet diese Frage genau? Warum erscheint diese Frage relevant bzw. warum interessiert sich die Forscherin gerade für diese Frage?

2. Auf welchen ontologischen Annahmen mit Blick auf die Beschaffenheit des Untersuchungsgegenstands basiert die Forschungsfrage?

3. Auf welchen epistemologischen Annahmen mit Blick auf Wahrheit und Wissenschaft basiert die Forschungsfrage?

4. Welches Material steht zur Analyse der zu untersuchenden Lebenspraxis zur Verfügung? Muss eventuell neues Material generiert werden (etwa durch Interviews)? Drückt sich im ausgewählten Material die zu untersuchende Lebenspraxis aus? Hat die Lebenspraxis im zur Analyse ausgewählten Pro-

5 Inzwischen unterscheide ich verschiedene Memo-Typen voneinander, die in den folgenden Abschnitten eingeführt und erörtert werden. Grundsätzlich handelt es sich bei einem Memo um einen Text, der einen Gedankengang ausbuchstabiert und der im Anschluss Eingang in eine klar geordnete Memo-Sammlung findet. Erst letzterer Vorgang macht aus einem Text ein Memo. Memos bleiben dabei jedoch , beweglich', können und sollen also immer weiter verändert werden. Das heißt, die Interpretation und deren Argumentationsgang bleiben fallibel. 
tokoll (möglichst deutliche) Spuren hinterlassen, deren Analyse die Beantwortung der aufgeworfenen Forschungsfrage begünstigen?

5. Falls das Zeitbudget der Forscherin nicht ausreicht, um alle protokollierten Spuren der zu untersuchenden sozialen Praxis zu analysieren, muss diesbezüglich eine Auswahl erfolgen. An welchen Kriterien orientiert sich diese Auswahl, wie wird die Auswahl des Materials begründet? Welche Sequenzen des Materials sind für die Analyse besonders geeignet?

6. Was versteht der Forscher unter Interpretation? Wie wird interpretiert? Welche Techniken kommen dabei zum Einsatz?

7. Wie wird der Forschungsprozess methodisch eingerichtet? Wie wird das Denken strukturiert, wie werden das Material und die sich einstellenden Interpretationen geordnet? Wie soll das analysierte Material geordnet werden?

8. Wie wird systematisch nach Zusammenhängen zwischen den einzelnen rekonstruierten Eigenschaften des Untersuchungsgegenstandes geforscht?

9. Wie werden die besonders einflussreichen, bedeutsamen, prägenden Eigenschaften des Untersuchungsgegenstands identifiziert?

10. Anhand welcher Kriterien wird über den Abschluss der Forschungssituation entschieden?

11. Anhand welcher Kriterien wird entschieden, welche Befunde veröffentlicht werden?

12. Auf welche Art und Weise wird die Präsentation der Befunde gestaltet? Wie erfolgt die Erörterung der Theorie und wie wird diese belegt? Wie wird die Nachprüfbarkeit bzw. die Nachvollziehbarkeit der Theorie gewährleistet?

13. Reflektieren die Forscher über Krisen und Grenzen der eigenen Vorgehensweise und werden Verbesserungsvorschläge mit Blick auf zukünftige Arbeiten formuliert?

\section{Die konkrete Vorgehensweise}

Ad 1) Wie lautet die Forschungsfrage? Nach welcher Art von Antwort verlangt diese Frage? Was bedeutet diese Frage genau? Warum erscheint diese Frage relevant bzw. warum interessiert sich der Forscher gerade für diese Frage?

Wie im ersten Abschnitt bereits kurz erörtert, lautet die von uns aufgeworfene Forschungsfrage wie folgt:

„,Welche grundlegenden Handlungsregeln und finalen Gründe bestimmen die Namibia-Politik der Fraktionen des Deutschen Bundestags und der Bundesregierung seit 1989/90 und welche Elemente von Kontinuität lassen sich bezüglich der darin repräsentierten außenpolitischen Kultur identifizieren? “ 
Der Forschungsprozess zielt darauf ab, gegen Ende eine in den Daten begründete Theorie der Namibia-Politik der Fraktionen des Deutschen Bundestags und der Bundesregierung vorlegen zu können und grundlegende Elemente der gegenwärtigen außenpolitischen Kultur Deutschlands zu rekonstruieren. Die bisherige Beschäftigung mit dieser und ähnlich gelagerten Frage(n) lässt mich vermuten, dass die außenpolitische Kultur des deutschen Nationalstaats z.B. eine erstaunliche, mehr als hundertjährige Kontinuität mit Blick auf ein Überlegenheitsgefühl - u.a., aber sicher nicht nur - gegenüber der namibischen Kultur und Gesellschaft aufweist. Nachdem ich mich bereits in der Vergangenheit intensiv mit deutscher Außenpolitik beschäftigt habe, vermute ich durch die Rekonstruktion der finalen Gründe der deutschen Namibia-Politik zu weitreichenden Erkenntnissen mit Blick auf grundlegende, besonders hartnäckig wirkmächtige Eigenschaften der außenpolitischen Kultur Deutschlands im Allgemeinen zu gelangen. Im Fragment spiegelt sich auch dort das Ganze - das Allgemeine im Besonderen.

Die Reflexionen zu den Fragen 2 (Auf welchen ontologischen Annahmen mit Blick auf die Beschaffenheit des Untersuchungsgegenstands basiert die Forschungsfrage?) und 3 (Auf welchen epistemologischen Annahmen mit Blick auf Wahrheit und Wissenschaft basiert die Forschungsfrage?) erfolgten bereits im Abschnitt 2 weiter oben. Daher fahre ich nun direkt fort mit Frage 4.

Ad 4) Welches Material steht zur Analyse der zu untersuchenden Lebenspraxis zur Verfügung? Muss eventuell neues Material generiert werden (etwa durch Interviews)? Drückt sich im ausgewählten Material die zu untersuchende Lebenspraxis aus? Hat die Lebenspraxis im zur Analyse ausgewählten Protokoll (möglichst deutliche) Spuren hinterlassen, deren Analyse die Beantwortung der aufgeworfenen Forschungsfrage begünstigen?

Mit Blick auf die Erforschung der deutschen Namibia-Politik steht reichhaltiges Material zur Verfügung. Neben den Protokollen von Sprechakten, wie z.B. den bedeutenden Parlamentsdebatten des Deutschen Bundestages zur deutschen Namibia-Politik in den Jahren 1989, 2004 und 2012 oder den anlässlich wechselseitiger Besuche gehaltenen Reden von Mitgliedern beider Parlamente und der Regierungen der beiden Staaten, bieten sich die Internet-Auftritte der beiden Botschaften und die zum Thema der deutsch-namibischen Beziehungen gehaltenen Ansprachen der beiden Botschafter als zusätzliche Quellen an. Ferner liegen eine große Zahl von Zeitungsbeiträgen der namibischen und deutschen Qualitätspresse sowie ein beachtlicher Korpus wissenschaftlicher Beiträge vor, die als Material zur Rekonstruktion der uns interessierenden Eigenschaften deutscher Namibia-Politik in Frage kommen. Das Durchführen 
von Interviews - etwa i) mit dem namibischen Botschafter in Deutschland, ii) Mitgliedern der jeweiligen deutsch-namibischen Freundschaftsgruppen in den beiden Parlamenten sowie iii) einschlägigen wissenschaftlichen Experten bzw. Journalisten - dürfte die Qualität der Rekonstruktion zusätzlich steigern.

Mit Blick auf die Frage nach geeignetem Material erscheint es mir wichtig, noch einmal zu wiederholen und zu konkretisieren, was ich bereits im ersten Abschnitt dieses Beitrags zum Thema Datenmaterial gesagt habe. Datenmaterial liegt in Form von Spuren des Untersuchungsgegenstandes vor. Die von uns untersuchte soziale Praxis, der untersuchte Prozess oder die untersuchten Diskurse und Strukturen kollektiven Handelns hinterlassen Spuren, die wir analysieren können. Bei diesen Spuren kann es sich natürlich um protokollierte Sprechakte handeln, genauso gut jedoch auch um andere Handlungstypen (Sie erinnern sich an das Beispiel eines Raketenangriffs). Für mich ist in diesem Zusammenhang auch die pragmatistische Maxime von Peirce von erheblicher Bedeutung. Wenn die Identität eines (sozialen) Gegenstandes davon abhängig ist, welche Eigenschaften wir diesem Gegenstand zuschreiben, dann stellen potentiell sämtliche Beschreibungen, die Dritte bezüglich unseres Forschungsgegenstandes vornehmen, Material für unsere Analyse zur Verfügung. Das bedeutet nicht weniger, als dass zwischen dem, was im Allgemeinen als ,Forschungsstand zum Untersuchungsgegenstand“ bezeichnet wird und unserem ,Untersuchungsgegenstand keine Differenz besteht. Wer eine Theorie über die Identität deutscher Außenpolitik formulieren möchte, kann auf den hierzu vorliegenden Forschungsstand nicht allein als Anregung zur Interpretation oder als potentielle Vergleichsfolie zurückgreifen, sondern muss die dort formulierten Hypothesen und Eigenschaftsbehauptungen als Material analysieren und interpretieren. Je nach Forschungsfrage mag es trotzdem relevant sein, die Selbstbeschreibungen deutscher Außenpolitik von den Fremdbeschreibungen zu unterscheiden, um bestimmte Beobachtungen anstellen und Unterscheidungen treffen zu können. Diese Unterscheidung sollte dann auch im Kodierbaum entsprechend berücksichtigt werden. Generell handelt es sich jedoch in beiden Fällen um Datenmaterial. In unserem Forschungsvorhaben stellen wir uns beispielsweise die Frage, welche grundlegenden Handlungsregeln und finalen Gründe die Namibia-Politik der Fraktionen des Deutschen Bundestags und der Bundesregierung seit 1989/90 bestimmen und welche Elemente von Kontinuität und Wandel sich bezüglich der darin repräsentierten außenpolitischen Kultur identifizieren lassen. Als Material können nun sämtliche Daten dienen, deren Analyse uns zu Wertungen und Erklärungen mit Blick auf unsere Forschungsfrage führen. Ganz gleich, ob es sich dabei um eine wissenschaftliche These bezüglich unseres Untersuchungsgegenstandes handelt, die ein brasilianischer Kollege vorlegt, der für ein neuseeländisches Forschungsinstitut tätig ist, um die Beschreibung eines deutschen Journalisten, der für eine russischsprachige Tageszeitung arbeitet, deren Herausgeber die Volksrepublik China ist, oder um eine vom ägyptischen Außenminister vorgetragene Kritik an 
der deutschen Außenpolitik, relevant ist für uns bloß, dass die formulierte Fremdbeschreibung sich auf unseren Untersuchungsgegenstand bezieht und bezüglich dessen Beschaffenheit Hypothesen formuliert. Statt nun jedoch diese Thesen einfach unkritisch zu übernehmen und für bare Münze zu nehmen, müssen wir die Fremdbeschreibungen genauso kritisch analysieren und interpretieren wie die Selbstbeschreibungen deutscher Außenpolitik.

Um jedoch Material identifizieren zu können, sind wir auf Kriterien angewiesen, die Material von Nicht-Matertial unterscheiden. Grundsätzlich gilt für mich alles als Material, aus dessen Analyse ich Hypothesen bezüglich der Beschaffenheit meines Untersuchungsgegenstandes gewinnen bzw. überprüfen und begründen kann.

\section{Ad 5) Falls das Zeitbudget der Forscherin nicht ausreicht, um alle protokollierten Spuren der zu untersuchenden sozialen Praxis zu analysieren, muss diesbezüglich eine Auswahl erfolgen. An welchen Kriterien orientiert sich diese Auswahl, wie wird die Auswahl des Materials begründet? Welche Sequenzen des Materials sind für die Analyse besonders geeignet?}

Insofern die Rekonstruktion von Handlungsregeln und finalen Gründen einer sozialen Praxis auf Grundlage der von ihr hinterlassenen Spuren und Wirkungen eine zeitintensive Tätigkeit darstellt, ist eine gute Auswahlstrategie bezüglich des analysierten Materials bedeutsam. Eine ideale Lösung für dieses Problem liegt jedoch bedauerlicher Weise nicht vor. Allerdings haben sich einige Auswahlfaktoren bewährt. Besonders wichtig ist für mich, mir stets der Forschungsfrage gewahr zu bleiben. Forschungsfragen können sich im Laufe eines Forschungsprozesses - zumal in den frühen Stadien - zwar fortentwickeln (ich halte dies sogar eher für die Regel als für eine zu vermeidende Ausnahme), doch gänzlich ohne Fokus lässt sich nicht forschen. Ich rate daher mit Blick auf die Auswahlentscheidungen des zu analysierenden Materials dringend dazu, sich im Vorfeld der Entscheidungen erneut möglichst klar zu vergegenwärtigen: Was will ich erforschen? Was interessiert mich an dieser Frage wirklich? Auf Grundlage der hierauf formulierten Antworten können Sie die Auswahl des Materials zielgerichteter durchführen. Außerdem sollten Sie Ihren ,gesunden Menschenverstand' einsetzen. Welche Auswahlentscheidungen erscheinen aus welchen Gründen vernünftig? Durch die Reflexion der ,guten Gründe', die für eine bestimmte Auswahlstrategie sprechen, ist bereits viel gewonnen. Indem Sie sich dazu zwingen, diese Auswahlkriterien zu verschriften und zu veröffentlichen und damit potentiell kritisierbar zu machen, heben Sie bereits die Qualität Ihrer Auswahlbemühungen, da Sie solcherart die imaginierte Kritik der potentiellen Leser Ihrer Studie vorwegnehmen und dies in die Begründungen einfließen lassen. Ich erstelle zu diesem 
Zweck eigens ein Dokument, in welchem ich meine Auswahlentscheidungen fortlaufend begründe. Außerdem sollten Sie sich intensiv mit Ihrem Forschungsgegenstand vertraut machen und systematisch das Kontextwissen steigern.

Für unser Namibia-Projekt ergeben sich daraus die folgenden Fragen: Was sind die bedeutenden früheren und gegenwärtigen Gegenstände, Probleme und Herausforderungen der deutsch-namibischen Beziehungen? Welche Ereignisse prägten und prägen diese Interaktion maßgeblich? Welche politischen Parteien, ökonomischen Interessengruppen und gesellschaftlichen Lager lassen sich diesbezüglich unterscheiden? In welchen Foren bzw. zu welchen Anlässen findet die Kommunikation zu diesen Themen statt?

Durch eine sorgfältige Annäherung an den Gegenstandsbereich erhalten wir wertvolle Einsichten mit Blick auf die Beschaffenheit des zu erforschenden Interaktionsverhältnisses, die unsere Datenauswahlentscheidung maßgeblich unterstützen. Weiterhin liegt es nahe, solche Spuren des Untersuchungsgegenstands zu analysieren, die seitens Dritter besonders starke oder zahlreiche Reaktionen hervorgerufen haben, wie beispielsweise die Rede der damaligen Bundesministerin für Entwicklung und Zusammenarbeit, Heidemarie Wieczorek-Zeul, bei den „Gedenkfeierlichkeiten der Herero-Aufstände" am 14. August 2004 in Okakarara (Wieczorek-Zeul 2004). Wenn einzelne (Sprech-)Handlungen innerhalb eines Diskurses eine besondere Resonanz erzeugt haben, dann nur deswegen, weil diesen eine besonders große Bedeutung zugesprochen worden ist. Selbstverständlich sollten wir uns dann für solche Handlungen ebenfalls besonders interessieren und eine eigene Interpretation derselben anfertigen.

Daneben greife ich auf zwei weitere Ideen zurück, die ich Anselm Strauss und Ulrich Oevermann verdanke: Das theoretical sampling-Verfahren und die pars-prototo-Idee. Bei Strauss erfolgt die Datenauswahl nicht abschließend bereits zu Beginn des Forschungsvorhabens, sondern der Forscher „entscheidet, welche Daten als nächstes zu erheben sind und wo er diese finden kann" auf Grundlage der aktuellen Situation des Forschungsprozesses (Strauss 1994: 70). Das Daten-Sample wird nicht entlang bereits im Vorfeld des Forschungsprozesses bestimmter Kriterien fixiert, sondern ergibt sich als Ergebnis des Versuchs, die im Laufe der Forschung formulierten vorläufigen Interpretationen mit solchem Material zu konfrontieren, von dem anzunehmen ist, dass es die bisherige Lesart möglichst maximal konzeptionell erweitern könne, um hierdurch immer erklärungskräftigere Hypothesen bezüglich der Beschaffenheit des Untersuchungsgegenstands als Elemente der entstehenden Theorie zu generieren. Es wird also von Fall zu Fall solches Material als nächster Datensatz ausgewählt, von dem zu hoffen ist, dass dieser im Blick auf die sich entwickelnden theoretischen Überlegungen zur Steigerung der konzeptionellen Repräsentativität (Strübing 2002: 335) beiträgt. 
Von Ulrich Oevermann übernehme ich das durchaus entlastende Argument, dessen Nähe zu dialektischen Grundprinzipien unübersehbar ist, dass die allgemeinsten finalen Gründe, also die grundlegendsten Handlungsregeln einer jeden sozialen Praxis, pars pro toto in allen Protokollen des Phänomens zum Ausdruck gelangen und folglich daraus rekonstruiert werden können. Das besonders Allgemeine findet sich in jedem Einzelnen. So nimmt Oevermann an, es reiche für ,die überwiegende Zahl der Fälle vollständig aus, vier kurze Segmente von maximal zwei Seiten“ aus einem wesentlich umfangreicheren Datensatz willkürlich auszuwählen, um eine „,hinreichende Fallrekonstruktion mit den Mitteln der Sequenzanalyse zu gewinnen"(Oevermann 2000: 97). Dies kann er meines Erachtens nur aufgrund der Annahme behaupten, dass sich die zentralen Konzepte und grundlegenden Handlungsregeln einer untersuchten sozialen Praxis aus jeder Stelle des Datenmaterials analysieren lassen. Wenn wir dann noch gelegentlich dem Zufall eine Chance geben und über den nächsten zu analysierenden Datensatz - im Anschluss an eine wohlüberlegte Vorauswahl - etwa durch eine Reihe von Würfelwürfen entscheiden, um wenigstens der Tendenz nach unwahrscheinlicher zu machen, dass wir nur solches Material analysieren, das sich als besonders geeignet erweist, unsere bisherigen Vorurteile zu bestätigen, rundet dies die Auswahlstrategie zusätzlich ab.

Bei umfänglichen Ereignissen wie einer längeren Rede, einer einstündigen Parlamentsdebatte oder einem ausführlichen Zeitungsartikel müssen - je nach Beschaffenheit der zeitlichen Ressourcen - ebenfalls Auswahlentscheidungen getroffen werden. In Anlehnung an die in seinen Seminaren ausgesprochenen Empfehlungen Oevermanns kann es sich anbieten, die oft sehr aussagekräftigen Eröffnungs- und Schlusspassagen eines Textes zu analysieren zuzüglich einiger weiterer Sequenzen aus dessen Hauptteil. Als Sequenz verstehe ich einen beliebig langen oder kurzen Text, der aus meiner Sicht eine Sinneinheit darstellt, die Aufschluss über die Handlungsregeln und finalen Gründe des Untersuchungsgegenstands ermöglicht.

Generell empfehle ich jedoch, jede Sequenz der zuvor derart sorgfältig ausgewählten Texte intensiv zu lesen und Sequenz für Sequenz darüber zu entscheiden, ob sich darin eine Handlungsregel oder ein finaler Grund ausdrückt, der zur Beantwortung der Forschungsfrage aufschlussreich sein könnte. Nur wenn dies der Fall ist, sollte die Sequenz zum Gegenstand einer ausführlichen Analyse gemacht werden.

\section{Ad 6) Was versteht der Forscher unter Interpretation? Wie wird interpretiert? Welche Techniken kommen dabei zum Einsatz?}

Mit Blick auf die Faktoren, die das Interpretieren bestimmen, ist Körperlichkeit wohl die bedeutendste und wohl am wenigsten beachtete. Wie vieles im Leben lässt sich das Interpretieren trainieren, so dass die Dauer, in der Sie ohne Pause interpretieren 
können, gesteigert werden kann. Ich selbst lese an Tagen des Interpretierens nach dem Aufstehen und vor dem Kodieren keine Zeitungen, konsumiere auch keine sonstigen Medien, lese keine Mails usw., da ich davon ausgehe, dass das Gehirn sich mit sämtlichen wahrgenommenen Informationen auch noch einige Zeit nach der sinnlichen Aufnahme beschäftigen muss. Unser Gehirn dürfte hier einem Großrechner ähneln, dessen Arbeitsintensität ebenfalls nachlässt, wenn im Hintergrund neben der aktuellen Anwendung noch zu viele Prozesse mit- und weiterlaufen. Außerdem nehme ich mir vor, niemals kürzer als zwei Stunden zu kodieren. Oft muss man sich in der ersten halben Stunde ein wenig zwingen, danach können sich jedoch richtige ,flows“ ergeben, die bis zu vier, fünf Stunden anhalten können. Mehr als fünf Stunden am Tag verbringe ich indes nicht mit Interpretationsarbeit, da die Qualität der Memos ansonsten deutlich nachlässt und erfahrungsgemäß das Arbeitspensum des Folgetages darunter leidet. Für mich hat es sich außerdem bewährt, zwischendurch kodierfreie Tage einzulegen, da in aller Regel das Zwischen- und Unbewusste an den formulierten Thesen weiterarbeiten und nach eintägiger Pause oft zahlreiche neue Gedanken zu jüngeren Memos vorliegen, die es nachzutragen gilt. Bei täglicher Kodierarbeit minimiert sich die Qualität dieser zwischenbewussten Nachbereitungs- und Abduktionsprozesse. Dies führt für mich zu einem Maximum von 18-20 Stunden aktiver Denk- und Schreibarbeit pro Woche, die durch intensive Lektüre und Exzerpieren noch reduziert wird. (Genauso wichtig ist für mich übrigens die Frage nach dem geeigneten Arbeitsraum).

Für mich hat es sich gerade bei weiter fortgeschrittenen Forschungsvorhaben bewährt, die Memosammlung von Zeit zu Zeit vollständig zu lesen und erneut zu vergegenwärtigen. Oft genug entstehen hierdurch auch ohne das Hinzuziehen neuen Materials neue Gedanken und natürlich unterstützt dies auch die Zuordnung des neuen Materials bzw. der neuen Memos beim Kodieren. Ich stelle mir hierbei die folgenden Fragen: Liegt hier eine neue, bislang unbekannte und unkodierte Eigenschaft vor oder handelt es sich beim Material bloß um einen weiteren Indikator für eine bereits rekonstruierte Eigenschaft?

Als ,Techniken“ greife ich auf die „künstliche Naivität“ (Oevermann 1996), das Mittel der Übertreibung und polemischen Zuspitzung, den sogenannten gesunden Menschenverstand, die Suche nach dem Verborgenen und die Frage nach den Interessen und der Macht zurück. Im Modus der künstlichen Naivität versuche ich Interpretationen zu entwickeln, die ich aufgrund meines Vorwissens möglicherweise eher für abwegig erachte. Das Mittel der Übertreibung und der polemischen Zuspitzung kann dazu beitragen, den eigentlichen Kern eines Gedankens überhaupt erst sichtbar werden zu lassen. Die besonders mutige, besonders aufschlussreiche und erklärungskräftige Hypothese kann sich zwischen den Zeilen des verfassten Memos versteckt halten und wird oft erst durch das Mittel der Übertreibung und Zuspitzung expliziert. Als gesunder Menschenverstand gilt mir die dominante, die naheliegende, 
,normale‘ Erklärung eines bestimmten Handelns oder Phänomens. Indem ich mich immer wieder daran erinnere, auch nach dem Nicht-Getanen und potentiell nur in Erwägung-Gezogenen (dem nicht-realisierten Handeln), dem Getanen, jedoch Verborgenen (dem Geheimen) und dem absichtlich Verzerrten (der Lüge) zu fragen, erhalte ich ebenfalls wichtige Interpretationsimpulse. Der Lüge, dem Geheimen und dem Nicht-Realisierten kommt man jeweils auf verschiedenen Wegen und in verschiedener Qualität auf die Spur. Mit Blick auf das Nicht-Realisierte lässt sich eine kontrafaktische Spekulation durchführen, die den Raum möglicher Alternativhandlungen auslotet. Welche Handlungsalternativen hätten bestanden? Was hätte außerdem potentiell gesagt oder getan werden können?

Das Geheime lässt sich nun zwar nicht beobachten, doch die Wirkungen, die geheimes Handeln hinterlässt, sind beobachtbar, was Rückschlüsse auf das Geheime ermöglicht. Als Paraphrase der oben erörterten pragmatistischen Maxime bedeutet dies: ,An Ihren Früchten sollt Ihr Sie erkennen'. Ein Typus der solcherart beobachtbaren Wirkungen von Geheimhandeln sind veröffentlichte Sprechakte (Dritter), die ein solches Geheimhandeln thematisieren. Dabei steht selbstverständlich nicht im Vordergrund, herausfinden zu wollen, was , wirklich' geschieht. Das Geheime ist das Geheime ist das Geheime. Wir werden der wahren - ob geheim oder nicht - Wirklichkeit ohnehin niemals gewahr. Doch es mag sinnvoll sein, davon auszugehen, dass gerade in der Weltpolitik einige Geheimhandlungen durchgeführt, einige davon von Dritten öffentlich gemacht, andere jedoch nicht thematisch werden. Eine mögliche Erklärung für Unerklärliches stellt daher immer das Geheime dar. Mit der Lüge beschäftigen wir uns ebenfalls besser nicht mit dem Ziel, diese erkennen und von der Wahrheit unterscheiden zu können. Dies können wir in den meisten Fällen nicht und nie mit vollkommener Gewissheit. Wir können jedoch festhalten, ob wir einen Sprechakt für eine Lüge halten und aus welchem Grund; wir können zur Kenntnis nehmen, wenn Diskursteilnehmer Dritte der Lüge bezichtigen und wir können uns daran erinnern, dass die Lüge nicht nur allzu menschlich und deshalb allgegenwärtig ist, sondern als Desinformation gar als Teil des nationalen Interesses, der Konzernoder NGO-Strategie gelten kann und es daher legitim sein sollte, das Gesagte mit den anderen Praktiken der Akteure zu vergleichen und sich zu fragen, ob das im Sprechhandeln angekündigte bzw. ex-post beschriebene Handeln mit anderen rekonstruierbaren Spuren des zu erklärenden Handelns konform geht oder ob wir hierbei Widersprüche feststellen können.

Abschließend möchte ich noch die Vor- und Nachteile des Interpretierens in Gruppen ansprechen. Eine Gruppe von Forschern kann eine größere Fülle von Interpretationsmöglichkeiten entwickeln, als dies einem einzelnen Forscher gelingen kann. Dies wirft jedoch unmittelbar das Problem der Kompetenz- und Machtverteilung auf. Wie organisiert eine Gruppe das gemeinsame Arbeiten? Wer protokolliert auf welche Art und Weise das Gedachte? Wie werden die Gedanken schriftlich fest- 
gehalten? Wie wird über die Plausibilität der Interpretation entschieden? Werden alle Interpretationen zunächst gleichberechtigt nebeneinander gestellt und verschriftet oder werden bestimmte Lesarten unterdrückt, als ,abwegig', ,verrückt ${ }^{6}$ oder ,unplausibel' unmittelbar aussortiert? Verfügen die Gruppenmitglieder über gleiche Mitsprache oder herrschen Machtasymmetrien? Vielfalt zu ordnen und zu verwalten ist außerdem sehr zeitintensiv. Ein klares ,Für-die-Gruppe-Argument', wie es gelegentlich vertreten wird, formuliere ich also nicht. Stattdessen greife ich auf das wachsweiche ,Kommt-auf-die-Gruppe-an'-Argument zurück und belasse es dabei.

\section{Ad 7) Wie wird der Forschungsprozess methodisch eingerichtet? Wie wird das Denken strukturiert, wie werden das Material und die sich einstellenden Interpretationen geordnet? Wie soll das analysierte Material geordnet werden?}

Um mein Denken zu unterstützen und Ordnung für mich zu gewährleisten, fertige ich sogenannte ,Kodierbäume“ als Word-Dokument an. ${ }^{6}$ Der Begriff des Kodierbaums bezieht sich auf den von Glaser und Strauss verwendeten Begriffs des ,Kodierens', an dem ich festhalte. Doch was bedeutet Kodieren und was ist ein Kodierbaum?

Um mit dem Kodieren beginnen zu können, müssen wir die oben genannten Fragen 1-5 bereits für uns beantwortet und Material für die Interpretation ausgewählt haben. Das ausgewählte Material wird nun Sequenz für Sequenz gelesen und zunächst danach befragt, ob sich hierin eine Eigenschaft (Handlungsregel, finale Gründe) des Untersuchungsgegenstandes ausdrückt, die zur Beantwortung der Forschungsfrage relevant zu sein scheint. Dazu dienen mir die folgenden Hilfsfragen, die ich mir immer wieder ins Gedächtnis rufe:

6 Obwohl der Einsatz spezifischer Analysesoftware (z.B. MAXQDA oder ATLAS.ti) unbestrittene Vorteile mit sich bringt (wie die problemlose Integration verschiedener Dateiformate, die farbige Markierung der in den Texten rekonstruierten Kategorien oder verschiedene hilfreiche Sortierfunktionen), bleibt bei mir ein Unbehagen mit Blick auf die offene Frage zurück, inwiefern der Einsatz dieser Hilfsmittel die Kreativität und theoretische Strenge des Interpreten dadurch unterminiert, als sie die Unmittelbarkeit des Sich-mit-denDaten-,Befassens“ noch stärker als etwa Windows Word reduzieren und die falsche Hoffnung erzeugen, die Software stelle die theoretischen Überlegungen, Ableitungen und Zusammenhänge beinahe von alleine her (Stern 2010: 120; Hesse-Biber 2010: 327/28). 
Hilfsfragen des Kodierens

- Auf welche Eigenschaften des Untersuchungsgegenstands ermöglicht die gelesene Sequenz Rückschlüsse bzw. welche Hypothesen bezüglich möglicher Eigenschaften des Gegenstands lassen sich aufgrund dieser Sequenz behaupten?

- Wurde bereits zuvor Material analysiert, das auf dieselbe oder gleiche Eigenschaft hinweist, so dass diesbezüglich bereits eine Hypothese formuliert wurde? Falls ja, legt die Sequenz eine Modifikation der bisherigen Hypothese nahe oder bestätigt sie diese? Wenn nein: Welche bislang noch unerforschten Eigenschaften des Untersuchungsgegenstands, die für die Beantwortung der Fragestellung relevant erscheinen, lassen sich aufgrund dieser Sequenz behaupten?

Ich verdeutliche dies an einem Beispiel: Am 17. Juni 2004 fand im Deutschen Bundestag zunächst eine kurze Debatte und dann eine Abstimmung über Drucksache 15/3329, Antrag der Fraktionen SPD und Bündnis 90/Die Grünen mit dem Titel „Zum Gedenken an die Opfer des Kolonialkrieges im damaligen Deutsch-Südwestafrika“ statt. Im Zuge dieser Debatte erklärte ein Abgeordneter als einziger Redner seiner Fraktion gleich zu Beginn seiner Ausführungen ${ }^{7}$ :

„Frau Präsidentin! Meine lieben Kolleginnen und Kollegen! Wir gedenken heute eines traurigen Ereignisses in der deutsch-namibischen Geschichte, nämlich des Aufstandes der Hereros und Nama gegen die Kolonialmacht Deutschland und dessen Niederschlagung vor 100 Jahren. Besonders schlimm war die billigende Hinnahme, dass ganze Bevölkerungsgruppen vernichtet worden sind. Deshalb dürfen wir die blutige Niederschlagung der Aufstände nicht vergessen“" (Eine-Fraktion 2004: 10427).

Aus meiner Sicht handelt es sich dabei um eine Sequenz, die uns bei der Beantwortung der von uns aufgeworfenen Forschungsfrage (s.o.) unterstützt. Aufgabe des weiteren Forschungsprozesses wird es nun sein, Hypothesen zu generieren und, falls sich diese später angesichts des Gesamtbestandes der Interpretationen als nicht plausibel entpuppen sollten, auch wieder fallen zu lassen. Ich bin jedoch davon überzeugt, dass es unnötig ist, sich bei der Formulierung von Hypothesen vorschnell selbst zu beschneiden. Eine in einem projektinternen Kodierbaum vorläufig formulierte Arbeitshypothese ,richtet keinen Schaden an'. Wir sollten uns allerdings gut überlegen,

7 Ich nenne den Namen des Abgeordneten und der Fraktion absichtlich nicht, da dies im Rahmen des vorliegenden Beitrags insofern redlicher sein dürfte, als das hier veröffentlichte Kodierbeispiel zu einem sehr frühen Zeitpunkt des derzeit noch laufenden Forschungsvorhabens entstanden ist und die hier formulierten Hypothesen noch nicht weiter überprüft werden konnten. Aller Wahrscheinlichkeit nach dürfte die fertige Studie - dann natürlich mit Blick auf das Material nicht mehr anonymisiert - gegen Ende 2014 vorliegen. 
welche Hypothesen wir nach Beendigung des Forschungsprozesses für veröffentlichungswürdig erachten und zur Diskussion stellen wollen. Bevor wir andere überzeugen wollen, sollten wir zunächst einmal selbst von unserer Theorie überzeugt sein.

Wichtig ist dabei das Verfassen ausführlicher Memos, die den einer Hypothese zugrundliegenden Gedanken- und Argumentationsgang wirklich konsistent und kohärent ausformulieren. Nur dieser Vorgang unterscheidet eine Hypothese von einer bloßen Meinungsbekundung. Das möglichst genaue Erklären einer Hypothese fördert die Nachvollziehbarkeit und ermöglicht somit besser begründeten Widerspruch - und hierin liegt wohl das zentrale Gütekriterium eines rekonstruktiv-interpretativen Verfahrens. Drei-Wort-Memos sind in diesem Sinne keine wirklichen Memos. Sie sind Alibis. Sie schaffen keine Begründungsketten, keine Argumente, bereiten keine theoretischen Überlegungen vor, sie sind banal und nichtssagend, oft duplizieren oder bestenfalls paraphrasieren sie bloß das Material. Vergegenwärtigen Sie sich beim Interpretieren bitte, dass Sie einer Forschungsfrage nachgehen, dass Sie etwas in Erfahrung bringen möchten, dass Sie neugierig sind, etwas Bestimmtes aufzuklären oder besser zu verstehen. Sie zielen mit Ihrer Arbeit auf das Schaffen einer Theorie, einer Interpretation, einer Erklärung bezüglich der von Ihnen aufgeworfenen gesellschaftlich und wissenschaftlich relevanten Fragestellung.

Bezüglich der oben stehenden Sequenz formuliere ich zunächst das folgende Sequenz-Memo ${ }^{8}$ :

Der Abgeordnete Z.Y. nimmt in dieser Debatte als einziger Abgeordneter seiner Fraktion teil, so dass angesichts der bekannten und allgemeinen Normen und Gepflogenheiten des Deutschen Bundestages, wie zum Beispiel des fraktionell-geschlossenen Abstimmungsverhaltens, davon ausgegangen werden kann, dass der Abgeordnete bemüht sein wird, eine Position zu vertreten, die der in seiner Fraktion vorherrschenden Position entspricht. Thema der Debatte ist der Antrag der Fraktionen SPD und Bündnis 90/Die Grünen mit dem Titel „Zum Gedenken an die Opfer des Kolonialkrieges im damaligen Deutsch-Südwestafrika" und die Frage, wie sich die einzelnen Fraktionen zu diesem Antrag in der folgenden Abstimmung verhalten werden. Zunächst fällt auf, dass im Gegensatz zum Titel des Antrags hier nicht von einem Kolonialkrieg, sondern von einem Aufstand der Herero und Nama gegen die Kolonialmacht Deutschland ausgegangen wird. Dies stellt einen erheblichen Unterschied dar, da es für einen Aufstand konstitutive Bedingung ist, dass ein festes Herrschaftsverhältnis besteht, gegen das sich die Aufständischen nunmehr auflehnen. Ob im Jahre 1903, dem Jahr des Ausbruchs der militärischen Konfrontation, sich eine solche Souveränität des Deutschen Reichs über seine Kolonie Deutsch-Südwestafrika

8 Weiter unten werde ich noch eingehender auf die Funktion des Memos zu sprechen kommen. Ich unterscheide inzwischen drei verschiedene Memotypen voneinander: i) das Sequenz-Memo, ii) das Kategorien-Memo und iii) das Indikator-Memo. 
jedoch ausgebildet hatte, ist völkerrechtlich umstritten (vgl. Jaguttis 2005). Stattdessen gehen die meisten Völkerrechtler von einem Kolonialkrieg aus, für den es wiederum konstitutiv ist, dass zwei getrennte Souveräne miteinander Krieg führen, um die Frage des Herrschaftsverhältnisses erst noch zu entscheiden. Die dort - im von uns damals als Deutsch-Südwestafrika bezeichneten Territorium - lebenden autochthonen Stämme hatten in ihrer Wahrnehmung zu keinem Zeitpunkt auf ihre Souveränität verzichtet, sondern ausschließlich einen sogenannten Schutzvertrag mit dem Deutschen Reich geschlossen (Jaguttis 2005: 133). Als sie gewahr wurden, dass die „Schutztruppe“ des Deutschen Reiches eigentlich zur Etablierung eines deutschen Herrschaftsverhältnisses entsandt worden war, das mit Unterdrückung und Ausbeutung ihrer Stämme einher gehen würde, kam es nicht zu einem Aufstand, sondern zu einem Krieg zweier völkerrechtlicher Subjekte, mit je eigenen Interessen. Dass der Abgeordnete 100 Jahre später stellvertretend für seine Fraktion den Begriff des ,Aufstands "wählt, um die damaligen Geschehnisse zu erklären, ist also enorm bedeutsam und voraussetzungsreich, da er die Verantwortung für die Folgen und Wirkungen der Konfrontation damit zumindest der Tendenz nach den „Aufständischen " zuschreibt, also aus den Opfern des ersten Völkermords der deutschen Geschichte, Täter macht, die sich gegen den bereits etablierten, also seitens der Herero und Nama angeblich zuvor anerkannten und daher legalen politischen Souverän zu Unrecht aufgelehnt hätten. Ganz folgerichtig wird dann in der vorliegenden Sequenz auch nicht die im Rahmen des Aufstandnarrativs durchaus als legitim erscheinende Niederschlagung als , schlimm “ bezeichnet, sondern bloß die billigende Hinnahme, dass hierbei ganze Bevölkerungsgruppen vernichtet worden seien. Und selbst hierbei belässt der Abgeordnete es im Vagen, ob die Verantwortung für diesen schlimmen Aspekt einer eigentlich legitimen Aufstandsniederschlagung, vom deutschen Nationalstaat zu verantworten sei, oder nicht vielleicht doch von den damaligen Anführern der Aufständischen selbst. Statt zu sagen: „Besonders schlimm war die billigende Hinnahme seitens der deutschen Kolonialtruppe, dass ganze Bevölkerungsgruppen durch die militärischen Operationen der deutschen Einheiten vernichtet worden sind“ sagt er ja bloß, dass „, die billigende Hinnahme, dass ganze Bevölkerungsgruppen vernichtet worden " seien, ,, besonders schlimm war". In dieser Formulierung bleibt letztlich unklar, wer das Subjekt dieser billigende Hinnahme gewesen ist, die deutschen Einheiten oder die Anführer der Herero und Nama. Um dies noch weiter zuzuspitzen, ließe sich auch der Satz „Wir gedenken heute eines traurigen Ereignisses in der deutsch-namibischen Geschichte, nämlich des Aufstandes der Hereros und Nama gegen die Kolonialmacht Deutschland und dessen Niederschlagung vor 100 Jahren " noch genauer analysieren. Wie genau wird der Anlass des Gedenkens durch den Deutschen Bundestag in dieser Sequenz begründet? Es handelt sich um ein „trauriges Ereignis“, gedacht werden soll nämlich ,, des Aufstandes der Herero und Nama gegen die Kolonialmacht Deutschland". Hier bezieht sich das Ge- 
denken auf den Aufstand als Ereignis und nicht auf die Opfer des Kolonialkriegs. Die Ereignisse werden also nicht nur anders bewertet (Kolonialkrieg vs. Aufstand), sondern auch der Referent des Gedenkens ist ein anderer (Opfer des Krieges vs. Aufstand). Diese Lesart unterstützt dann zusätzlich die Interpretation, dass der Aufstand „, an sich“ ein ,trauriges" Ereignis darstellt. Nicht weniger als genau das wird ja explizit gesagt. Es gilt der Fraktion im Jahr 2004 als trauriges Ereignis, dass es im Jahr 1903 zu einem Aufstand gegen die deutsche Kolonialmacht gekommen ist, in dessen Verlauf es dann (besonders im Jahr 1904) zur ,,blutigen Niederschlagung“ gekommen ist. Ich leite daraus die folgenden Hypothesen ab: Die Analyse des Materials legt nahe,

i) dass die Fraktion davon ausgeht, dass im Jahr 1903 ein legales Herrschaftsverhältnis des Deutschen Reichs über Deutsch-Südwestafrika etabliert war;

ii) dass die Fraktion im Jahr 2004 vermeidet, zu erklären, welche Gründe zu diesem „Aufstand" führten und wer dafür die Verantwortung trägt;

iii) dass die Fraktion durch die Reproduktion des Aufstandnarrativs eine Lesart nahelegt, die besagt, dass die Verantwortung für die damaligen Geschehnisse zumindest der Tendenz nach den „Auftständischen “ selbst zukomme;

iv) dass die Fraktion, indem sie den Aufstand gegen das als etabliert angenommene Herrschaftsverhältnis als ,,trauriges Ereignis “ bezeichnet, signalisiert, dass keine guten Gründe bestanden, sich gegen dieses Herrschaftsverhältnis aufzulehnen v) dass die Fraktion es ablehnt, der Opfer der besonders grauenhaften deutschen Kriegsführung zu gedenken und stattdessen lieber dem Aufstand ,, als traurigem Ereignis" gedenken möchte.

$\mathrm{Zu}$ den einzelnen, hier aufgrund der Analyse einer einzigen Sequenz natürlich nur vorläufig und zaghaft gebildeten Hypothesen, überlege ich mir nun i) einen Titel, nummeriere ii) die Hypothesen und füge iii) die Sequenz, deren Interpretation zum Formulieren der These geführt hat, als Beleg, als Indikator für diese These hinzu und ordne iv) der Sequenz per Word-Kommentarfunktion das oben stehende Memo zu, so dass die Gedankengänge und die Begründungsstruktur, die zur Formulierung der These geführt haben, unmittelbar vorliegen und von Dritten nachvollzogen werden können (vgl. hierzu auch das unten stehende Schaubild). In Anlehnung an Strauss bezeichne ich eine von mir behauptete Eigenschaft des Gegenstands als Kategorie und kürze dies mit , $\mathrm{K}^{\circ} \mathrm{ab}$. Durch die weitere Analyse werden die meisten der formulierten Hypothesen verworfen bzw. fortlaufend verfeinert, modifiziert, jedenfalls immer weiter überprüft und mit Blick auf ihre Plausibilität hoffentlich verbessert. So entstehen die ersten Zweige meines Kodierbaums. 


\section{Kodierbaum des Namibia-Projektes Kodierbaum bezüglich der XYZ-Fraktion}

\section{Verzeichnis aller analysierten Sequenzen}

Sequenz 1: „Frau Präsidentin! Meine lieben Kolleginnen und Kollegen! Wir gedenken heute eines traurigen Ereignisses in der deutsch-namibischen $\mathrm{Ge}-$ schichte, nämlich des Auftstandes der Hereros und Nama gegen die Kolonialmacht Deutschland und dessen Niederschlagung vor 100 Jahren. Besonders schlimm war die billigende Hinnahme, dass ganze Bevölkerungsgruppen vernichtet worden sind. Deshalb dürfen wir die blutige Niederschlagung der Aufstände nicht vergessen“" (Eine-Fraktion 2004: 10427, Sequenz 1).

Sammlung der rekonstruierten Eigenschaften

K101 „Glaube an die Legalität deutscher Kolonialherrschaft“

Eigenschafts-Memo:

In dieser Kategorie sammle ich sämtliche von mir formulierten Hypothesen bezüglich der Überzeugungen der Fraktion mit Blick auf die Legalität der deutschen Kolonialherrschaft in Deutsch-Südwestafrika. Eine grundlegende erste These lautet, dass die Fraktion davon ausgeht, dass im Jahr 1903 ein legales Herrschaftsverhältnis des Deutschen Reichs über Deutsch-Südwestafrika etabliert war. Eine Ursache dieser Überzeugung könnte darin wurzeln, dass die Fraktion jener völkerrechtlichen Position anhängt, die davon ausgeht, dass zum damaligen Zeitpunkt nur europäische Nationalstaaten als Völkerrechtsubjekte anerkannt waren, denen es legaler Weise zustand vermeintlich nicht-souveräne Territorien zum Teil des eigenen Herrschaftsraum zu erklären (Jaguttis 2005). Dies könnte darauf hinweisen, dass die Fraktion generell darum bemüht ist, das damalige wie auch das gegenwärtige außenpolitische Handeln Deutschlands als völkerrechtskonform zu beschreiben. Ein solches Narrativ des ,,das Recht war und ist auf unserer Seite " könnte sowohl mit Blick auf mögliche Reparationsforderungen der Nachkommen der vom Völkermord betroffenen Herero und Nama als Argument von Bedeutung sein, als auch mit Blick auf die offene Frage der Landreform im heutigen Namibia, also zum Schutz der Besitzverhältnisse der deutschstämmigen Großgrundbesitzer und Farmer. Den Einen könnte gesagt werden, dass der Völkermord kein Verbrechen dargestellt habe-zumal das damalige Recht diesen Begriff noch nicht kannte, eine weitere beliebte Argumentationsfigur im deutschen Diskurs zur Reparationsfrage -, sondern vielmehr der Aufstand nicht rechtens gewesen sei. Und den heute landlosen Nachkommen der Ermordeten ließe sich sagen, dass das Land damals rechtskonform erworben worden und eine Enteignung Unrecht sei. 


\section{Sammlung der Indikatoren für K101}

„Frau Präsidentin! Meine lieben Kolleginnen und Kollegen! Wir gedenken heute eines traurigen Ereignisses in der deutsch-namibischen Geschichte, nämlich des Aufstandes der Hereros und Nama gegen die Kolonialmacht Deutschland und dessen Niederschlagung vor 100 Jahren. Besonders schlimm war die billigende Hinnahme, dass ganze Bevölkerungsgruppen vernichtet worden sind. Deshalb dürfen wir die blutige Niederschlagung der Aufstände nicht vergessen“" (Eine-Fraktion 2004: 10427, Sequenz 1).

Sequenz-Memo:

Im Original-Kodierbaum füge ich an dieser Stelle das oben stehende lange Memo ein, verzichte hier aber aus Platzgründen darauf. Am Ende des Memos stehen fünf Hypothesen (s.o.). Aus diesen Thesen leite ich fünf Eigenschaften der außenpolitischen Kultur der analysierten Fraktion ab (vgl. K101 bis 105), füge jeweils dieselbe Sequenz als Indikator (als Beleg) für die fünf verschiedenen Thesen ein und stütze die einzelne These ab, indem ich das vollständige Sequenz-Memo jeweils an den Indikator als Indikator-Memo anhänge. Im vorliegenden Fall wird das - zugegeben besonders lang ausgefallene Memo gleich sechsmal in den Kodierbaum integriert (zu Beginn der Sequenz zugeordnet und im weiteren Verlauf fünfmal als Indikator-Memo). Alternativ schneide ich jene Elemente des Sequenz-Memos aus, die zur Erörterung und Abstützung der gerade thematischen Eigenschaft irrelevant sind, und fertige fünf spezifisch zugeschnittene Indikator-Memos an. Letzteres ist sicherlich die optimalere Vorgehensweise, ist jedoch mit einem zusätzlichen Arbeitsschritt verbunden und entsprechend zeitintensiv, da viele Überlegungen aufeinander verweisen, sich wechselseitig abstützen und daher nicht ohne Weiteres ,ausgeschnitten' werden können. Ein Vorteil besteht sicherlich darin, dass die je spezifisch zugeschnittenen Indikator-Memos deutlich kürzer ausfallen als das ursprüngliche Sequenz-Memo und der Kodierbaum solcherart vor unnötigem Wildwuchs befreit wird und durch die Reduktion des Volumens übersichtlicher bleibt.

\section{Quellenverzeichnis}

Eine-Fraktion 2004: Deutscher Bundestag 2004: Plenarprotokoll 15/114, Deutscher Bundestag, Stenographischer Bericht der 114. Sitzung vom 17. Juni 2004, Berlin.

Grundsätzlich sollten Kodierbäume meines Erachtens so strukturiert sein, dass Sie die Forscherin bei ihrer Aufgabe unterstützen, systematisch über den Untersuchungsgegenstand nachzudenken und diese Gedanken zu ordnen. Ich arbeite zu diesem Zweck mit Microsoft Word und greife dabei auf die Hyperlink-, Index- und Inhaltsverzeichnisfunktion zurück. Ich markiere Sequenzen, die ich als Indikatoren für zwei 
oder mehr verschiedene Kategorien verwende, indem ich sie mit verschiedenen Farben versehe (schwarz, wenn die Sequenz nur als Indikator einer Eigenschaft zugeordnet wird, blau, wenn sie zwei Eigenschaften als Indikator dient usw.). Die Memos füge ich den Sequenzen per Kommentarfunktion hinzu. Und selbstverständlich werden die Sequenzen mit einer eindeutigen Zitation - ,Eine-Fraktion 2004: 10427` und der Kodierbaum mit einem fortlaufend aktualisierten Quellenverzeichnis versehen. Ferner erstelle ich ein Dokumente und Quellen übergreifendes Gesamtverzeichnis sämtlicher von mir analysierten Sequenzen, um jeder im Laufe des Forschungsprozesses analysierten Sequenz eine eindeutige Sequenznummer zuteilen zu können. Dieses Verzeichnis stelle ich dem Kodierbaum voran. Dies hat den Vorteil, dass ich in den einzelnen Memos klare Bezüge zwischen verschiedenen Sequenzen herstellen kann, also eindeutig auf Sequenzen verweisen kann, ohne befürchten zu müssen, dass sich diese Verweise noch einmal verschieben („Bereits das zu Sequenz 76 formulierte Memo reflektiert die Frage des Zusammenhangs zwischen den Konzepten ,Verantwortung' und ,Selbstüberhöhung “"). Wenn ich das Kodieren einer neuen Sequenz beginne, füge ich die neu zu analysierende Sequenz zunächst an das Ende dieses Verzeichnisses ein und beginne mit meiner Interpretationsarbeit. Das zu dieser Sequenz formulierte Memo füge ich an dieser Stelle per Kommentarfunktion der Sequenz hinzu. Danach kopiere ich die Sequenz (samt daran per Kommentarfunktion angehängtem Memo) und ordne diese als Indikator der jeweiligen Kategorie bzw. den Kategorien zu, für deren Hypothesen diese Sequenz als Beleg gilt. Dabei dient sehr häufig ein und dieselbe Sequenz als Indikator für verschiedene Kategorien und wird entsprechend häufig kopiert (inklusive der beigefügten Indikator-Memos, wenn diese nicht kategorienspezifisch zugeschnitten werden). Falls die Sequenz als weiterer Indikator (Beleg) für eine bereits gebildete Kategorie fungiert, füge ich die Sequenz am Ende der Indikatorensammlung dieser Kategorie ein. Falls aufgrund der Interpretation dieser Sequenz eine oder mehrere neue Hypothesen formuliert wurden, überlege ich mir einen erklärungskräftigen Titel für diese Kategorie, vergebe die nächste freie Kategorie-Nummer und füge die Sequenz als ersten Indikator in die Indikatorensammlung dieser neugebildeten Kategorie ein.

Abschließend füge ich jeder Kategorienüberschrift ein Memo hinzu, das erklärt, warum diese Kategorie gebildet wurde, welche Eigenschaft des Gegenstandes diese Kategorie erklärt bzw. rekonstruiert. Dieses Kategorien-Memo kann im Laufe des fortschreitenden Forschungsprozesses als Ort dienen, um die darunter in den Indikator-Memos ausformulierten Thesen bezüglich der in dieser Kategorie erklärten Zusammenhänge und Eigenschaften zusammenzufassen und kohärent zu beschreiben. Die Kategorien-Memos werden fortlaufend ergänzt und modifiziert. Sie fassen einerseits die Gehalte der empirisch gesättigten Hypothesen noch einmal komprimiert zusammen und können gleichzeitig als Ort dienen, um über mögliche Ursachen und Wirkungen der behaupteten Eigenschaften weiterführende Hypothesen zu for- 
mulieren, die sich bestenfalls auf andere bereits rekonstruierte Eigenschaften stützen und auf diese verweisen. Letztlich erörtert also dieses Kategorien-Memo die bezüglich der behaupteten Eigenschaft des Gegenstands hin angestellten Hypothesen und liefert eine konsistente und kohärente Argumentationskette bezüglich der vorgelegten Interpretation und greift hierbei auf die in den darunter subsumierten IndikatorMemos festgehaltenen Überlegungen zurück. Hierbei lässt sich eine gewisse Redundanz zwischen Kategorien-, Sequenz- und Indikator-Memos nicht vermeiden.

Der Vorteil besteht nun darin, dass die auf einzelne Sequenzen bezogenen Gedanken (der Sequenz- und Indikator-Memos) nun von jenen Eigenschaftsbehauptungen zu unterscheiden sind, die aufgrund der Gesamtschau aller unter eine Kategorie subsumierten Sequenzen (als Indikatoren) und Memos entstanden sind. Die oben stehende Beispiel-Sequenz würde also zunächst katalogisiert und mit einer eindeutigen Zitation und Sequenznummer versehen (etwa: Eine-Fraktion 2004: Seq. 1). Danach würde das hierzu angefertigte Memo z.B. per Kommentarfunktion als Sequenz-Memo eingefügt werden. Daraufhin würden die fünf neu gebildeten Kategorien K101 ,Legalität deutscher Kolonialherrschaft‘ bis K105 ,Ablehnung des Gedenkens an die Opfer der Herero und Nama' angelegt und fünfmal dieselbe analysierte Sequenz jeweils als Indikator darunter eingefügt werden. Das identische Memo würde ebenfalls allen fünf Indikatoren als Indikator-Memo hinzugefügt werden bzw. entsprechend kategorienspezifisch zugeschnitten werden. In diesem Zusammenhang ist es mir wichtig, darauf hinzuweisen, dass sich im Laufe der Zeit - durch die Analyse weiterer Sequenzen und darin gefundener weiterer Indikatoren bzw. durch neue aus dem Kontextwissen gespeiste Irritationen und daran anknüpfende neue Überlegungen - auch die Texte bereits zuvor formulierter Kategorien-, Sequenz- und IndikatorMemos fortlaufend verändern können. Dasselbe gilt natürlich auch für die Titel der Kategorien. Nun mag es anfangs durchaus frustrierend sein, wenn alle Überlegungen noch derart fluide sind. Doch machen Sie sich bitte bewusst, dass sämtliche Modifikationen dazu beitragen, Ihre im Entstehen befindliche Theorie zu verbessern. Beginnend mit der Analyse der ersten ausgewählten Sequenz, dem ersten gefertigten Memo, der ersten formulierten Hypothese und der ersten daraus abgeleiteten Kategorie entsteht eine neue Theorie.

Es ist nicht verwunderlich, wenn sich bei Ihnen inzwischen einige Fragen angesammelt haben. Ich versuche im Weiteren einige dieser Fragen zu antizipieren und zu beantworten.

Aus dem oben stehenden Beispiel-Sequenz-Memo geht hervor, wie bedeutsam Kontextwissen für das Anfertigen von Interpretationen ist. Ohne die vorherige Lektüre von Jaguttis 2005 hätte ich die Sequenz weniger intensiv interpretiert. Indem mir die Argumente von Jaguttis jedoch bekannt waren, konnte ich in meiner eigenen Interpretation darauf zurückgreifen. Ich bin davon überzeugt, dass jede Interpretation auf Vorwissen zurückgreifen muss. Wir erklären uns ein Ereignis, eine Handlung 
durch Rückgriff auf unser Wissen. Wir wollen das Phänomen auf den Begriff bringen, prädizieren, wir wollen es uns erklären. Ich gehe davon aus, dass jede Interpretation zugleich eine Erklärung darstellt, die auf einer Beschreibung basiert. Wir stellen zunächst analytische Beobachtungen an, stellen Hypothesen bezüglich der Eigenschaften des beobachteten Prozesses oder Gegenstands auf und versuchen in einem weiteren, dann bereits synthetischen Schritt, Zusammenhänge zwischen diesen Eigenschaften zu erkennen, um schließlich darauf aufbauend besonders grundlegende Mechanismen, Eigenschaften und Zusammenhänge des Untersuchungsgegenstands zu identifizieren.

Dieser Dreischritt von Analyse, Synthese und Erklärung der Eigenschaften des Gegenstands ist zentral für mein Verständnis rekonstruktiver Sozialforschung: Wir analysieren die Eigenschaften des Untersuchungsgegenstands und bilden dabei Kategorien - Eigenschaftsbehauptungen - ersten Abstraktionsgrades. Diese Hypothesen basieren unmittelbar auf der Interpretation des ausgewählten empirischen Materials. Im Zuge der Synthese suchen wir nun nach Zusammenhängen zwischen den bereits rekonstruierten Eigenschaften und formulieren diesbezüglich auf unseren Gegenstand bezogene Eigenschaftsbehauptungen zweiten Abstraktionsgrades - zweiten Abstraktionsgrades deswegen, weil die Hypothesen, welche die Eigenschaften dieser synthetischen Kategorien konstituieren, in aller Regel nicht unmittelbar im Zuge der Materialanalyse entstehen, sondern durch die Suche nach Zusammenhängen zwischen den bereits schriftlich fixierten Hypothesen und Kategorien ersten Grades. Ich illustriere dies kurz. Aus dem Zusammenspiel der fünf oben formulierten Hypothesen mit einer weiteren im Laufe des Forschungsprozesses entstandenen Hypothese (K106) ließe sich eine weit abstraktere Hypothese (zweiten Abstraktionsgrads) formulieren, die so lauten könnte9:

Insofern die Fraktion davon ausgeht, dass im Jahr 1903 ein legales Herrschaftsverhältnis des Deutschen Reichs über Deutsch-Südwestafrika etabliert war (K101), sie zugleich den Aufstand gegen das als etabliert angenommene Herrschaftsverhältnis als "trauriges Ereignis" bezeichnet und damit signalisiert, dass keine guten Gründe bestanden, sich gegen dieses Herrschaftsverhältnis aufzulehnen (K104) und dies gleichzeitig nahelegt, dass die Verantwortung für die damaligen Geschehnisse zumindest der Tendenz nach den „Auftständischen“ selbst zukomme (K105), lässt sich die Hypothese formulieren, dass die Fraktion die deutsche Kolonialherrschaft über das damalige Deutsch-Südwestafrika nicht allein als legal, sondern auch als legitim erachtet. Ich bilde diesbezüglich die Kategorie zweiten Abstraktionsgrades ,K201 Legitimität deutscher Kolonialherrschaft:

9 Ich schreibe, so lauten könnte', da ich diese nun zum ersten Mal formuliere und noch nicht weiter überprüft habe. 
Aus welchen Gründen die Fraktion diese Legitimität genau ableitet, muss hier noch als offen gelten. Unter Rückgriff auf die bereits an anderer Stelle formulierte These, dass die Fraktion einen Rückgang des gegenwärtigen Engagements der Bundesrepublik bzw. der deutschstämmigen Farmer in Namibia für äußerst nachteilhaft für die ökonomische und politische Stabilität Namibias erachtet (K106 ,Besonders fruchtbarer Einfluss Deutschlands auf das heutige Namibia'), ließe sich unter Rückgriff auf die im Forschungsstand vertretene These vom sich hartnäckig haltenden Mythos des segensreichen Einflusses der deutschen Kulturträger auf seine Kolonien (vgl. Böhlke-Itzen 2005: 109) jedoch bereits eine erste, vorläufige Hypothese für eine mögliche Erklärung dieser Position formulieren: Die Fraktion ist davon überzeugt, dass die deutsche Herrschaft über Deutsch-Südwestafrika legitim gewesen ist, weil die deutschen Kolonisatoren auf die autochthonen Völker einen besonders heilsamen Einfluss ausgeübt haben. Ich bilde daraus die Unter-Kategorie zweiten Grades ,K201 a Besonders heilsamer Einfluss der deutschen Kolonisatoren begründet die Legitimität der deutschen Kolonialherrschaft:

In den sogenannten Unterkategorien unterscheide ich verschiedene potentielle Elemente einer Erklärung der behaupteten Eigenschaft. Solche Unterkategorien können sowohl für K100er- als auch K200er-Kategorien formuliert werden und auch ihrerseits weitere Unterkategorien erhalten, die diese noch präziser erklären.

Selbstverständlich stehen diese neuen Hypothesen zweiten Abstraktionsgrades nämlich i) dass die Fraktion die deutsche Kolonialherrschaft über das damalige Deutsch-Südwestafrika nicht allein als legal, sondern auch als legitim erachtet (K201) und ii) dass die Fraktion davon überzeugt ist, dass die deutsche Herrschaft über Deutsch-Südwestafrika legitim gewesen sei, weil die deutschen Kolonisatoren auf die autochthonen Völker einen besonders heilsamen Einfluss ausgeübt haben (K201 a) - auf noch vergleichsweise ,tönernen“ Füßen.

Aus diesem Grund ist es so wichtig zu betonen, dass sämtliche im Laufe des Forschungsprozesses formulierten Hypothesen stets fallibel bleiben, also zu jedem möglichen Zeitpunkt verworfen werden können. Hypothesen stellen nicht den Versuch dar, letztgültige Wahrheiten und Naturgesetzlichkeiten zu formulieren. Sie müssen scheitern können, sonst sind es keine Hypothesen. Wir sollten offen bleiben für Kritik, selbst an unseren besonders liebgewonnenen Überlegungen. Dies bedeutet auch, dass der von uns angefertigte Kodierbaum stets beweglich bleiben sollte. Sie werden immer wieder mühselig geformte Kategorien verwerfen müssen, während andere Hypothesen in Vergessenheit geraten, weil die dort rekonstruierte Eigenschaft des Untersuchungsgegenstands in keiner weiteren Sequenz thematisch wird. Kategorien können aufgelöst und ineinander integriert werden, weil Sie nach einigen Wochen feststellen, dass Sie zwei Kategorien unterschiedlichen Namens angelegt haben, die sich zueinander letztlich redundant verhalten. Nichts ist in Stein gemeißelt. 
Wie unsagbar schwierig muss es angesichts dessen früher gewesen sein, mit analogen Medien wie hand- oder maschinebeschriebenen Karteikarten zu arbeiten? Waren frühere Forschergenerationen, die noch alles analog bearbeiten und sortieren mussten, möglicherweise weniger schnell dazu bereit, Hypothesen aufzugeben, zu modifizieren und ineinander zu integrieren, weil dies einen enormen Arbeitsaufwand bedeutete? Oder waren die in den Memos festgehaltenen Beobachtungen, Argumente und Kausalketten von vorneherein von höherer Qualität, weil sie potentiell für eine längere Zeit von Bestand bleiben sollten? Oder trifft beides zu?

\section{Ad 8) Wie wird systematisch nach Zusammenhängen zwischen den einzelnen rekonstruierten Eigenschaften des Untersuchungsgegenstandes geforscht?}

Je weiter Ihr Forschungsvorhaben voranschreitet, umso mehr Eigenschaften ersten Unmittelbarkeitsgrades (K100er-Kategorien) liegen Ihnen vor. Wie oben bereits kurz erwähnt, ist es ab einer bestimmten Menge an vorliegenden Hypothesen und theoretischen Überlegungen notwendig, von Zeit zu Zeit den gesamten Bestand der bereits formulierten Memos und rekonstruierten Eigenschaften durch eine zusammenhängende Lektüre aller Memos zu vergegenwärtigen. Indem Sie das Gesamt Ihrer theoretischen Sätze aktualisieren, bereiten Sie das Feld für jenen Vorgang, den Charles Sanders Peirce in Anlehnung an Aristoteles Abduktion genannt hat. Neben Deduktion, dem Schluss auf künftige Fälle auf Grundlage einer Hypothese, und Induktion, dem Schluss von einem Fall auf eine bestehende Hypothese, stellt Peirce das abduktive Schließen, verstanden als Moment der Formulierung einer neuen Hypothese. ${ }^{10}$ In diesem Sinne führen wir immer, wenn wir eine neue Hypothese formulieren, einen abduktiven Schluss durch. Die neu gewonnene Hypothese dient als Grundlage wei-

10 Unter Abduktion versteht Peirce (unter Rückgriff auf Aristoteles) die Formulierung einer neuen Hypothese aufgrund neuer Erfahrungen. Peirce war zu dem Schluss gelangt, dass weder Induktion noch Deduktion dazu geeignet seien, neue Hypothesen zu kreieren (Peirce [1903] 1965: 89-90 (5.144)). Denn aus dem induktiven Verfahren allein resultiert noch keine modifizierte Erklärung einer bestehenden bzw. die erstmalige Formulierung einer neuen Hypothese: „Abduction is the process of forming an explanatory hypothesis. It is the only logical operation which introduces any new idea; for induction does nothing but determine a value, and deduction merely evolves the necessary consequences of a pure hypothesis. Deduction proves that something must be; Induction shows that something is actually operative; Abduction merely suggests that something may be. Its only justification is that from its suggestion deduction can draw a prediction which can be tested by induction, and that, if we are ever to learn anything or to understand phenomena at all, it must be by abduction that this is to be brought about" (Peirce [1903] 1965: 106 (5.171)). 
terer Deduktionen und wird durch induktive Schlüsse permanent überprüft. Alle drei Schlussverfahren laufen gleichzeitig ab, bilden eine Einheit und sind nicht voneinander zu trennen.

Wenn ich an dieser Stelle den Stellenwert der Abduktion besonders betone, dann heißt dies nicht, dass im Zuge des Kodierens ersten Unmittelbarkeitsgrades (K100erKategorien) nicht ebenfalls bereits abduktive Schlüsse formuliert werden. Ich möchte nur darauf hinweisen, dass bei der systematischen Suche nach Zusammenhängen zwischen bereits formulierten Hypothesen und durch das Aktualisieren und Vergegenwärtigen dieser Hypothesen das Feld für weitere Abduktionen bereitet wird. Ich unterscheide dabei generell das abduktive Schließen in drei verschiedene Unmittelbarkeitsgrade. Abduktive Schlüsse ersten Unmittelbarkeitsgrades resultieren in meinem Verständnis aus der Betrachtung und Interpretation von Datenmaterial. Ich beobachte ein (Sprech-)Handeln, ich denke darüber nach, ich interpretiere es und formuliere unter Bezugnahme auf das Beobachtete meine Sinnzuschreibung. Alle K100er-Kategorien und die darunter gesammelten Hypothesen fallen hierunter. Davon unterscheide ich solche abduktiven Schlüsse, die sich nicht unmittelbar durch die Beobachtung von Datenmaterial einstellen, sondern die sich aus dem Nebeneinander, dem Zusammenspiel, dem Vergleich bzw. der Kontrastierung bereits formulierter Hypothesen ergeben. Häufig entsteht die neu gebildete Hypothese dann nicht auf Grundlage einer weiteren analysierten Datensequenz, sondern aufgrund der permanenten Suche nach möglichen Zusammenhängen zwischen bereits behaupteten Eigenschaften des Untersuchungsgegenstands. Diese abduktiven Schlüsse zweiten Unmittelbarkeitsgrades bilden die K200er-Kategorien. Sobald eine solche, auf anderen Hypothesen basierende Hypothese formuliert wurde, ist es leicht möglich, Sequenzen zu identifizieren, die als Indikator für diese Hypothese fungieren können. Allerdings ist es denkbar und kommt auch vor, dass sich keine weiteren Indikatoren für eine K200er-Kategorie finden lassen. Dies ist auch gar nicht nötig, da die Hypothese auf Vorhypothesen (K100er) zurückgreift, die jeweils bereits in Daten begründet wurden und bis zuletzt nicht verworfen worden sind. K200er- und K100er-Kategorien unterscheiden sich in meiner Lesart also entlang der Komplexität der formulierten Hypothesen, da im Falle der K200er-Kategorien zumindest zwei Vorhypothesen miteinander verbunden werden.

\section{Ad 9) Wie werden die besonders einflussreichen, bedeutsamen, prägenden Eigenschaften des Untersuchungsgegenstands identifiziert?}

In Anlehnung an Anselm Strauss und dessen Idee der Schlüsselkategorie unterscheide ich außerdem noch K300er-Kategorien von den beiden anderen Kategorietypen. Für Strauss müssen Schlüsselkategorien „zentral sein“, sich „mühelos in Bezug setzen“ 
lassen zu anderen Kategorien und „häufig im Datenmaterial vorkommen“ (Strauss 1994: 67). Ich habe an anderer Stelle die These formuliert, dass die Suche nach der einen Schlüsselkategorie bei Strauss die Funktion der Identifikation der einen unabhängigen Variable in klassischen Forschungsdesigns ersetzt (Roos 2010: 106ff.). Aus diesem Grund werbe ich dafür, nicht nach der einen Schlüsselkategorie fahnden zu wollen, sondern halte es für naheliegend, dass eine größere Zahl von Schlüsseleigenschaften vorliegen kann. Ganz ähnlich wie Strauss schreibe ich einer solchen Eigenschaft des Untersuchungsgegenstandes wiederum die Eigenschaft zu, besonders erklärungskräftig für eine große Zahl von Kategorien des ersten und zweiten Unmittelbarkeitsgrades zu sein. Durch das Formulieren dieser Hypothesen dritten Unmittelbarkeitsgrades wird die Erklärungskraft zahlreicher Elemente der angefertigten Theorie, wie der Theorie insgesamt, gesteigert. Die Theorie - so ließe sich sagen - ,macht viel mehr Sinn', entwickelt durch diese Schlüsselkategorien eine größere Kohärenz und kann entlang dieser Schlüsselkategorien Stück für Stück, Hypothese für Hypothese entfaltet werden.

Um ein Beispiel aus einem abgeschlossenen Forschungsvorhaben (Roos 2010, 2012) zu geben, behaupte ich, dass die von mir bezüglich der grundlegenden Handlungsregeln deutscher Außenpolitik aufgestellte Theorie maßgeblich von den Schlüsselkategorien ,Kampf zwischen realistischen und idealistischen Überzeugungen " und ,Heilsame Wirkung des überlegenen deutschen Wesens auf seine internationale Umwelt" bestimmt wird. Keine der beiden Schlüsselkategorien lässt sich aufgrund einzelner Sequenzen unmittelbar am Material ,beweisen'. Angesichts der in den Daten begründeten Rekonstruktionsarbeit lassen sich diese Eigenschaften deutscher Außenpolitik jedoch rekonstruieren und meines Erachtens wird das Handeln deutscher Außenpolitik aufgrund dieser Hypothesen deutlich besser erklärbar.

Es leuchtet ein, dass die Identifikation von Schlüsselkategorien tendenziell eher am Ende eines Forschungsvorhabens steht und nicht am Anfang. Gerade im Kontext von Forschungsvorhaben geringerer Dauer und Intensität, wie sie zum Beispiel im Zuge von einsemestrigen universitären Seminarkontexten häufig anzutreffen sind, sollten Sie nicht verzweifeln, wenn Sie Schwierigkeiten damit haben, solche Schlüsselkategorien zu entwickeln. Besser Sie verzichten darauf, als dass Sie schlecht abgesicherte Schlüsseleigenschaften behaupten, die nicht plausibel sind.

\section{Ad 10) Anhand welcher Kriterien wird über den Abschluss der Forschungssituation entschieden?}

Anselm Strauss führte mit Blick auf die Frage nach dem Abschluss der Forschungssituation das Kriterium der empirischen Sättigung ein, der gemäß eine Kategorie dann als ,saturiert' gelten könne, wenn während des Kodierens keine neuen Eigenschaften, 
Dimensionen, Bedingungen, Handlungen/Interaktionen oder Konsequenzen gefunden werden können:

„Saturation is more a matter of reaching the point in the research where collecting additional data seems counterproductive...or, as is sometimes the situation, the researcher runs out of time, money, or both" (Strauss/Corbin 1998: 136; Strauss 1994: 49).

Forschungssituationen werden letztlich zu keinem Zeitpunkt endgültig abgeschlossen. Wenn Sie eine Abschlussarbeit, eine Seminararbeit, ein Lehrforschungsprojekt oder eine Dissertation abschließen, dann heißt dies ja bloß, dass Sie Hypothesen bezüglich eines Gegenstandes veröffentlichen, wie Sie diese zum Zeitpunkt der Niederschrift des Manuskripts vertreten. Insofern wir glücklicherweise immer weiter Erfahrungen sammeln, die untersuchte Praxis uns auch zukünftig Wirkungen hinterlässt, die von Dritten interpretiert werden, wird sich unser Denken über diesen Gegenstand immer zu fortentwickeln. Eine wissenschaftliche Veröffentlichung erscheint aus dieser Perspektive besehen nur in den seltensten Fällen als für ,die Ewigkeit' verfasst, sondern stellt nicht mehr als die Auskopplung einer Momentaufnahme dar, um der wissenschaftlichen Gemeinschaft die eigenen Überlegungen zu einem bestimmten Zeitpunkt mitzuteilen, diese damit in die Debatte einzuspeisen, angreifbar zu machen und durch die geäußerte Kritik die eigenen Lernprozesse noch zu beschleunigen. Niemand hält Sie davon ab, zu einem späteren Zeitpunkt, eine überarbeitete Theorie zum selben Gegenstand zu veröffentlichen. Bevor Sie jedoch eine Theorie publizieren, sollten Sie wenigstens selbst von deren Plausibilität überzeugt sein.

\section{Ad 11) Anhand welcher Kriterien wird entschieden, welche Befunde veröffentlicht werden?}

Ich veröffentliche nur solche Hypothesen, von denen ich mich selbst überzeugt habe. Ich imaginiere dabei die wissenschaftliche Gemeinschaft, die Vertreter anderer, abweichender Lesarten und nehme deren Reaktionen auf meine Theorie und meine eventuell vom Forschungsstand abweichenden Interpretationen vorweg. Ich halte es für ein sinnvolles Kriterium, nur solche Argumente zu veröffentlichen, von denen ich sagen kann, dass sie bis zuletzt plausibel geblieben sind und sich in die Theorie kohärent einfügen. Auch publiziere ich bloß solche Hypothesen, die ich zugleich allen potentiell daran interessierten, imaginierbaren signifikanten Dritten gegenüber verteidigen könnte. Eine Hypothese über die Beschaffenheit deutscher Europapolitik zu formulieren, die ich nicht bereit wäre, auch einem Vertreter des Auswärtigen Amtes gegenüber zu vertreten, der sich hauptberuflich mit der Gestaltung deutscher Europapolitik befasst, würde ich nicht veröffentlichen, wenn ich allzu große Zweifel an 
deren Plausibilität hegte und ich deren Zustandekommen nur unzureichend erklären könnte. All das bedeutet natürlich nur, dass auch zu dieser Frage keine Letztentscheidungskriterien formuliert werden können. Dies mag unbefriedigend sein. Ich füge dennoch die These hinzu, dass über diese Frage vor allen Dingen die charakterliche Disposition der Forschenden entscheidet.

Ad 12) Auf welche Art und Weise wird die Präsentation der Befunde gestaltet? Wie erfolgt die Erörterung der Theorie und wie wird diese belegt? Wie wird die Nachprüfbarkeit bzw. die Nachvollziehbarkeit der Theorie gewährleistet?

Sobald Sie den Entschluss gefasst haben, Ihre Theorie zu veröffentlichen, stehen Sie vor einem weiteren nicht zu unterschätzenden Problem: Wie lassen sich die Befunde auf angemessene Art und Weise präsentieren? Am Ende meines Dissertationsprojektes zur Entwicklung deutscher Außenpolitik von 1989/90 bis ins Jahr 2007 umfasste der Kodierbaum inklusive der Memos über 1000 Textseiten. Kein Verlag veröffentlicht einen solchen Text-Korpus als Teil des gedruckten Buches. Daher entschied ich mich dazu, den Kodierbaum auf CD zu brennen und im Anhang zu veröffentlichen bzw. auf meiner universitären Mitarbeiter-Homepage zu veröffentlichen, um die intersubjektive Nachvollziehbarkeit der aufeinander verweisenden Thesen en detail - zumindest potentiell - zu ermöglichen. Im veröffentlichten Buch präsentierte ich meine Befunde, fügte diesen Befunden meine Begründungsketten hinzu, erklärte also aufgrund welcher Beobachtungen und Zusammenhänge ich zu den veröffentlichten Hypothesen bezüglich meines Forschungsstandes gelangt war und wählte eine illustrative Belegstrategie. Das heißt, aus dem vollständigen Kodierbaum wählte ich jene Sequenzen zum Zwecke des illustrativen Belegens der Thesen aus, von denen ich annahm, dass sich die von mir unterstellten Eigenschaften darin besonders eindeutig zeigen ließen und auch von LeserInnen nachvollzogen werden konnten, die sich nicht knapp fünf Jahre mit dem gesamten analysierten Material auseinandergesetzt haben.

Wir können die Leser unserer Theorien bei ihren Bemühungen, unsere Theorien nachzuvollziehen, durch eine wohldurchdachte Argumentationsfolge maßgeblich unterstützen. In einem Buch oder Aufsatz müssen Sie sich entscheiden, in welcher Reihenfolge Sie die Elemente Ihrer Theorie präsentieren. Insofern die einzelnen Elemente der Theorie aufeinander verweisen, kommen Sie in aller Regel nicht daran vorbei, mit Hypothesen und theoretischen Sätzen zu beginnen, die Ihre volle Plausibilität und Erklärungskraft erst entfalten können, wenn alle Elemente der Theorie entfaltet werden konnten und vom Leser nachvollzogen wurden. Auch hier existiert wohl kein idealer Weg, um diese Herausforderung zu meistern. Sich dieser Heraus- 
forderung bewusst zu sein, richtet jedenfalls keinen Schaden an. Hier führen wohl verschiedene Wege zum Ziel. Sie können zunächst die Schlüsselkategorien erörtern und darauf hinweisen, dass der Leser über deren Plausibilität bitte erst entscheiden möge, sobald er den gesamten Text gelesen hat. Oder Sie beginnen mit der Rekonstruktion einzelner Eigenschaften des Untersuchungsgegenstands und erklären darauf aufbauend immer mehr Zusammenhänge zwischen den einzelnen Eigenschaften, bis Sie schließlich die Schlüsselkategorien präsentieren. Oder Sie präsentieren kurz die Schlüsselkategorien, bevor Sie danach die für deren Herleitung notwendigen Elemente der Theorie einführen, um gegen Ende die Schlüsselkategorie ausführlich zu erklären. Wichtig ist meines Erachtens bloß, die Leser über den eingeschlagenen Kurs und das dabei verfolgte Ziel zu Beginn des Ergebniskapitels Ihrer Arbeit zu informieren.

\section{Ad 13) Reflektieren die Forscher über Krisen und Grenzen der eigenen Vorgehensweise und werden Verbesserungsvorschläge mit Blick auf zukünftige Arbeiten formuliert?}

Insofern für eine rekonstruktiv-interpretative Verfahrensweise das regelmäßige Verfassen von Memos zentral ist, liegt es nahe, auch die Krisen und Problemlösungen mit Blick auf methodologische und methodische Fragen fortlaufend zu protokollieren. Ich greife hierzu auf ein separates Dokument zurück, in dem ich meine Abduktionen und Glaubenskrisen bezüglich dieser Fragen protokolliere. Dies entlastet während des Forschungsprozesses, treibt die Problemlösung voran, da sich ausbuchstabierte Zweifel zielgerichteter bearbeiten lassen als zwischenbewusste Zweifel, und liefert die Textgrundlage für eine abschließende Reflexion der eigenen Vorgehensweise in der Abschlusspublikation. Wenn es möglich sein sollte, extensive Anhänge zu veröffentlichen, ließe sich das gesamte solcherart im Laufe des Forschungsprozesses gefertigte Methoden-Memo anfügen.

Der hier vorliegende Beitrag stellt insofern Resultat und Element dieser fortlaufenden Reflexionsarbeit dar. 


\section{Literatur}

Bauer, Harry/Brighi, Elisabetta (Hrsg.) 2009: Pragmatism in International Relations, Abingdon.

Baumann, Rainer 2006: Der Wandel des deutschen Multilateralismus, Baden-Baden.

Böhlke-Itzen, Janntje 2005: Die bundesdeutsche Diskussion und die Reparationsfrage, in: Melber, Henning (Hrsg.): Genozid und Gedenken, Frankfurt a. M., 103-19.

Bryant, Antony/Charmaz, Kathy (Hrsg.) 2010: The Sage Handbook of Grounded Theory, London.

Dewey, John [1927] 1991: The Public and its Problems, Athens, OH.

Dewey, John [1910] 1997: How We Think, Boston, MA.

Eine-Fraktion 2004: Deutscher Bundestag 2004: Plenarprotokoll 15/114, Deutscher Bundestag, Stenographischer Bericht der 114. Sitzung vom 17. Juni 2004, Berlin.

Franke, Ulrich 2010: Die Nato nach 1989. Das Rätsel ihres Fortbestandes, Wiesbaden.

Franke, Ulrich/Roos, Ulrich 2010: Actor, Structure, Process: Transcending the State Personhood Debate by Means of a Pragmatist Ontological Model for International Relations Theory, Review of International Studies 36: 4, 1057-77.

Franke, Ulrich/Weber, Ralph 2012: At the Papini Hotel: On Pragmatism in the Study of International Relations, European Journal of International Relations 18: 4, 669-91.

Friedrichs, Jörg/Kratochwil, Friedrich 2009: On Acting and Knowing: How Pragmatism Can Advance International Relations Research and Methodology, International Organization 63: 4, 701-31.

Glaser, Barney/Strauss, Anselm 1967: The Discovery of Grounded Theory: Strategies for Qualitative Research, Chicago.

Hellmann, Gunther (Hrsg.) 2006: Germany's EU Policy in Asylum and Defence: De-Europeanization by Default? Basingstoke.

Hellmann, Gunther 2009: Beliefs as Rules for Action: Pragmatism as a Theory of Thought and Action, International Studies Review 11: 3, 638-62.

Hellmann, Gunther 2010: Pragmatismus, in: Masala, Carlo/Sauer, Frank/Wilhelm, Andreas (Hrsg.): Handbuch der Internationalen Politik, Wiesbaden, 148-81.

Hellmann, Gunther/Roos, Ulrich 2007: Das deutsche Streben nach einem ständigen Sitz im UN-Sicherheitsrat. Analyse eines Irrwegs und Skizzen eines Auswegs, INEF-Report 92, Duisburg/Essen.

Hesse-Biber, Sharlene Nagy 2010: Teaching Grounded Theory, in: Bryant, Antony/Charmaz, Kathy (Hrsg.): The Sage Handbook of Grounded Theory, London, 311-38.

Hofferberth, Matthias 2012: Multinationale Unternehmen in den internationalen Beziehungen. Zur Kontingenz von Bedeutung und Rolle sozialer Akteure, Frankfurt a. M., unv. Dissertation.

Hulswit, Menno 2002: From Cause to Causation. A Peircean Perspective, Dordrecht.

Jaguttis, Malte 2005: Koloniales Unrecht im Völkerrecht der Gegenwart, in: Melber, Henning (Hrsg.): Genozid und Gedenken, Frankfurt a. M, 121-40. 
Jasper, Ursula 2014: The Politics of Nuclear Non-Proliferation: A Pragmatist Framework for Analysis, Milton Park.

Mead, George Herbert [posthum 1934] 1974: Mind, Self and Society: from the Standpoint of a Social Behaviorist, Chicago, IL.

Oevermann, Ulrich 1991: Genetischer Strukturalismus und das sozialwissenschaftliche Problem der Erklärung der Entstehung des Neuen, in: Müller-Doohm, Stefan (Hrsg.): Jenseits der Utopie. Theoriekritik der Gegenwart, Frankfurt a. M., 267-336.

Oevermann, Ulrich 1996: Konzeptualisierung von Anwendungsmöglichkeiten und praktischen Arbeitsfeldern der objektiven Hermeneutik (Manifest der objektiven hermeneutischen Sozialforschung), Frankfurt a. M., unv. Manuskript.

Oevermann, Ulrich 2000: Die Methode der Fallrekonstruktion in der Grundlagenforschung sowie der klinischen und pädagogischen Praxis, in: Kraimer, Klaus (Hrsg.): Die Fallrekonstruktion, Frankfurt a. M., 58-153.

Peirce, Charles Sanders [1878] 1992: Hot to Make Our Ideas Clear, in: Houser, Nathan/Kloesel, Christian (Hrsg.): The Essential Peirce: Selected Philosophical Writings, Vol. I, 1867-1893, Bloomington, IN, 124-41.

Peirce, Charles Sanders [1903] 1965: Lectures on Pragmatism, in: Hartshorne, Charles/Weiss, Paul (Hrsg.): Collected Papers of Charles Sanders Peirce, Volume V: Pragmatism and Pragmaticism, 3. Auflage, Cambridge, MA, 14-212.

Peirce, Charles Sanders [1905] 1998: What Pragmatism Is, in: The Peirce Edition Project (Hrsg.): The Essential Peirce: Selected Philosophical Writings, Vol. II, 1893-1913, Bloomington, IN, 331-45.

Roos, Ulrich 2010: Deutsche Außenpolitik. Eine Rekonstruktion der grundlegenden Handlungsregeln, Wiesbaden.

Roos, Ulrich 2012: Deutsche Außenpolitik nach der Vereinigung. Zwischen ernüchtertem Idealismus und realpolitischem Weltordnungsstreben, Zeitschrift für Internationale Beziehungen 19: 2, 7-40.

Stern, Phyliss Noerager 2010: On Solid Ground: Essential Properties for Growing Grounded Theory, in: Bryant, Antony/Charmaz, Kathy (Hrsg.): The Sage Handbook of Grounded Theory, London, 114-26.

Strauss, Anselm 1987: Qualitative Analysis for Social Scientists, Cambridge, MA.

Strauss, Anselm 1993: Continual Permutations of Action, New York, NY.

Strauss, Anselm 1994: Grundlagen qualitativer Sozialforschung: Datenanalyse und Theoriebildung in der empirischen und soziologischen Forschung, München.

Strauss, Anselm 2004 a: Methodologische Grundlagen der Grounded Theory, in: Strübing, Jörg/Schnettler, Bernt (Hrsg.): Methodologie interpretativer Sozialforschung, Konstanz, 427-51.

Strauss, Anselm 2004 b: Anselm Strauss im Interview, in: Legewie, Heiner/Schervier-Legewie, Barbara, in: Forum Qualitative Sozialforschung 5: 3, Artikel 22, http://www.qualitative-research.net/index.php/fqs/article/view/562/1219; 30.9.2013.

Strauss, Anselm/Corbin, Juliet 1998: Basics of Qualitative Research: Techniques and Procedures for Developing Grounded Theory, London. 
Strübing, Jörg 2002: Just Do It? Zum Konzept der Herstellung und Sicherung von Qualität in grounded-theory-basierten Forschungsarbeiten, Kölner Zeitschrift für Soziologie und Sozialpsychologie 54: 2, 318-42.

Strübing, Jörg 2004: Grounded Theory. Zur sozialtheoretischen und epistemologischen Fundierung des Verfahrens der empirisch begründeten Theoriebildung, Wiesbaden.

Wagner, Wolfgang/Baumann, Rainer/Bösche, Monika/Hellmann, Gunther 2006: German Foreign Policy in Europe: An Interactionist Framework of Analysis, in: Hellmann, Gunther (Hrsg.): Germany's EU Policy on Asylum and Defence. De-Europeanization by Default?, Basingstoke, 1-28.

Wieczorek-Zeul, Heidemarie 2004: Rede von Bundesministerin Heidemarie Wieczorek-Zeul bei den Gedenkfeierlichkeiten der Herero-Aufstände am 14. August 2004 in Okakarara. 